\title{
Modelling flow-enhanced crystallisation during fused filament fabrication of semi-crystalline polymer melts
}

\author{
C. McIlroy, R. S. Graham \\ School of Mathematical Sciences, University of Nottingham, Nottingham, UK, NG7
}

\begin{abstract}
Achieving better control in fused filament fabrication (FFF) relies on a molecular understanding of how thermoplastic printing materials behave during the printing process. For semi-crystalline polymers, the ultimate crystal morphology and how it develops during cooling is crucial to determining part properties. Here crystallisation kinetics are added to a previously-developed model, which contains a molecularly-aware constitutive equation to describe polymer stretch and orientation during typical non-isothermal FFF flow, and conditions under which flow-enhanced nucleation occurs due to residual stretch are revealed. Flow-enhanced nucleation leads to accelerated crystallisation times at the surface of a deposited filament, whilst the bulk of the filament is governed by slower quiescent kinetics. The predicted time to $10 \%$ crystallinity, $t_{10}$, is in quantitative agreement with in-situ Raman spectroscopy measurements of polycaprolactone (PCL). The model highlights important features not captured by a single measurement of $t_{10}$. In particular, the crystal morphology varies cross-sectionally, with smaller spherulites forming in an outer skin layer, explaining features observed in full transient crystallisation measurements. Finally, exploitation of flow-enhanced crystallisation is proposed as a mechanism to increase weld strength at the interface between deposited filaments.
\end{abstract}

Keywords:

Fused filament fabrication; semi-crystalline polymer melt; flow-enhanced crystallisation; polycaprolactone

\section{Introduction}

Fused filament fabrication (FFF) is an extrusion-based approach to additive manufacturing (AM). Compared to other AM techniques such as laser-sintering and stereolithography, the advantage of FFF is the durable highperformance thermoplastic materials it uses [1]. FFF remains the cheapest technique for printing thermoplastic parts and is mostly popular with hobbyists. Advanced application of FFF is limited due to considerable variability in mechanical properties, which depend on the numerous processing conditions, as well as the material rheology (for some examples, see $[2,3,4,5,6,7,8,9])$.

The mechanical properties of a printed part are ultimately determined by the micro-structural configuration of the molecules upon solidification. Thermoplastics are made up of large, flexible polymer molecules that entangle with each other to form a polymer 'melt'. Quantitative predictions of part properties must account for how the polymers deform and relax during the flow and subsequent cooling, as well as any crystallisation processes. Employing molecularly-aware constitutive models is a key step to developing a fundamental understanding of the relationships between printing parameters and micro-structure, and ultimately improving and advancing FFF so that its potential can be exploited for main-stream processes.

The non-linear viscoelastic behaviour of molten poly- mers during flow is well documented and remains an active area of research (for example, see [10, 11]). During FFF, both the shear flow within the nozzle and the velocity gradients induced by the deposition process, where the material exits the nozzle and is deposited onto a build plate, can significantly deform the polymer micro-structure. In particular, McIlroy \& Olmsted [12] postulate an idealised deposition process where the deposit shape is prescribed, the velocity profile is determined by conservation of mass and the polymers are advected by velocity gradients. Under these assumptions, the polymers are stretched and aligned with the flow direction, and there is distinct gradient in the polymer micro-structure across the filament due to the deposition flow. A number of other models have also been derived to describe the deposition process [13, 14], although these are not molecularly-aware.

After deposition the melt cools, solidifies and bonds (welds) with adjoining material so that the structure of the final object consists of a number of partially-welded filaments. Shear-stress governed de-bonding along the weld lines is known to be a key failure mechanism [15]. Successful welding of the deposited filaments relies on interdiffusion and entanglement of the polymers across layerlayer interfaces via reptation [16]. Reptation is thermally driven, and is affected by the melt anisotropy [17, 18, 19], as well as any developing crystal morphology [20, 21, 22].

Consequently, non-isothermality introduces extra com- 


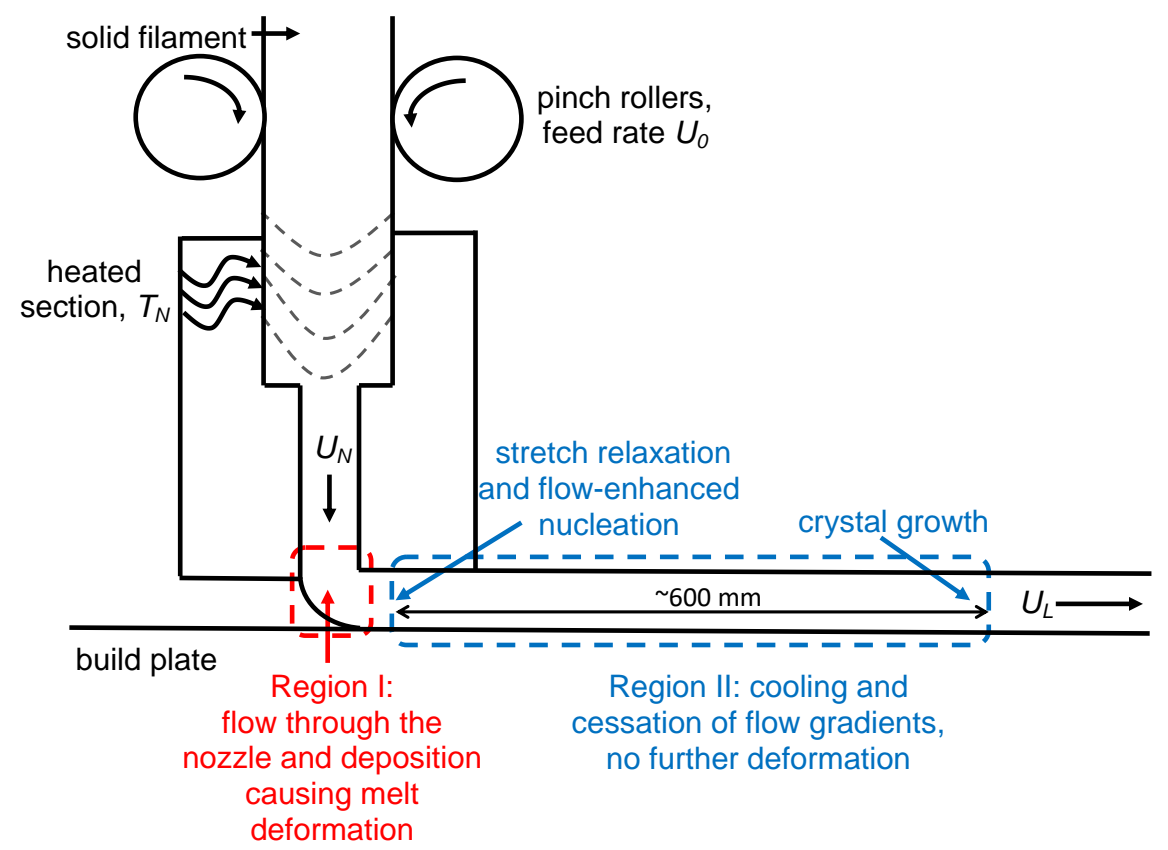

Figure 1: Schematic of the fused filament fabrication process modelled in this paper.

plexity to the polymer dynamics, with non-crystalline melts approaching a glass transition upon cooling to $T_{g}$, whilst semi-crystalline melts will nucleate and crystallise at temperatures below the melting temperature $T_{M}$ before transitioning to a glassy state at $T_{g}<T_{M}$; at $T_{g}$ diffusion, and therefore crystallisation, is arrested. Both classes of polymer are widely used in FFF, with semi-crystalline materials having the important advantage of biodegradability, essential for regenerative medical applications in tissue engineering [23, 24].

It is suggested that inter-diffusion should precede crystallisation and the glass transition to ensure good adhesion and weld strength [25]. Optimising the printing conditions to establish interfacial molecular diffusion and mechanically effective interfaces has recently been investigated experimentally for poly-lactic acid [26]. Also contributing to the ultimate strength of a printed part is the final degree of crystallinity achieved during cooling. It has recently been shown that the crystallinity of a printed part can vary with print temperature and layer thickness [27], and even the colour of the feedstock material [28].

Yet to be considered in FFF models is the effect of 'flow-enhanced' crystallisation - a now well-known phenomenon (for example, see the review by Graham [29] and references therein); if the polymer backbone is stretched at the onset of nucleation, then the nucleation rate is enhanced. This flow-enhanced nucleation speeds up the crystallisation process and leads to reduced crystallisation times compared to quiescent kinetics. Recent in-situ Raman spectroscopy experiments suggest that flow-enhanced crystallisation can occur under certain FFF printing conditions [30].

In our previous work we predicted the micro-structure of the weld region and diffusive inter-penetration depths, in the absence of crystallisation, and inferred a weld fracture toughness for a range of printing parameters [31]. Here we introduce crystallisation kinetics to this model to investigate how the stretch induced by the FFF process can enhance nucleation and consequently reduce the time taken for crystallisation. Understanding how the FFF flow affects crystallisation is the foundation to our future work understanding inter-diffusion dynamics and weld strength achieved with semi-crystalline materials.

We consider the (semi-crystalline) printing material polycaprolactone (PCL). PCL is often used for printing tissue scaffolds [32]. Furthermore, infrared thermography and Raman spectroscopy have recently been used to measure the temperature and crystallisation kinetics of this material during FFF [30]. For this polymer, room temperature is $T_{a} \gg T_{g}$ so that the glass transition is not approached under typical conditions.

This paper is organised as follows. Sec. 2 describes previously-published in-situ crystallisation measurements and observations of flow-enhanced crystallisation during FFF of PCL [30]; we derive a simple test to identify conditions under which flow-enhanced crystallisation will not occur and describe our model for quiescent crystallisation. In Sec. 3 we introduce our flow-enhanced crystallisation model, which requires only a single extra parameter. Predictions of the crystal morphology and crystallisation times in a deposited filament are presented in Sec. 4 alongside the experimental data from Ref. [30]. Finally, we discuss the interplay between cooling, polymer stretch and nucleation in Sec. 5 and identify a regime where accelerated crystallisation saturates. In the conclusion we hypothesize how flow-enhanced crystallisation may lead to 


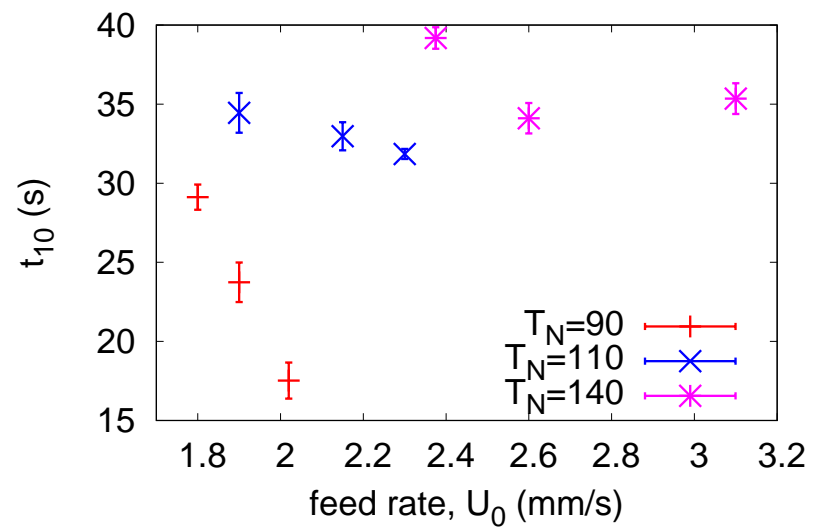

Figure 2: Data replicated from Ref. [30]. Time to $10 \%$ crystallisation $t_{10}$ measured using a Raman probe directed at the top surface of a deposited filament of PCL.

increased weld strength.

\section{General concepts}

\subsection{Crystallisation measurements and observations dur- ing FFF}

Recent experiments by Kotula et al. [30] use in-situ Raman spectroscopy to probe crystallisation rates of PCL during FFF. The PCL material used in Ref. [30] has number-averaged molecular weight $M_{n}=57.1$ and weightaveraged molecular weight $M_{w}=96.7 \mathrm{~kg} / \mathrm{mol}$. The FFF process is modified slightly to allow for the crystallisation measurements: a single filament of PCL is deposited onto a moving build plate from a fixed nozzle (radius $R_{N}=0.25$ $\mathrm{mm}$ ) and wound onto a take up wheel. The (radiallyaveraged) extrusion speed at the nozzle exit $U_{N}$ is matched to the speed of the moving build plate $U_{L}$. The gap between the nozzle and the build plate is set to $H=2 R_{N}$, which is kept at the ambient temperature $T_{a}=20^{\circ} \mathrm{C}$.

In general, this process can be divided into two regions, as shown in Fig. 1:

- Region I: the flow region. Here the melt flows through the hot nozzle $\left(T=T_{N}\right)$ and is deposited onto a build plate. The polymers are deformed due to velocity gradients in the flow.

- Region II: the cooling region. Here the melt cools and the polymer deformation relaxes in the absence of velocity gradients. Once $T<T_{M}$, the polymer will nucleate and spherulites will begin to grow.

If there is residual polymer stretch at the onset of nucleation in Region II, then crystallisation will be enhanced [29].

In Ref. [30], a Raman probe measures the crystal fraction at the top surface of the deposited filament as a function of distance from the nozzle exit $y$. This is then converted to a time measurement via $t=y / U_{N}$. The print temperature is varied between $T_{N}=90-140^{\circ} \mathrm{C}$ and the

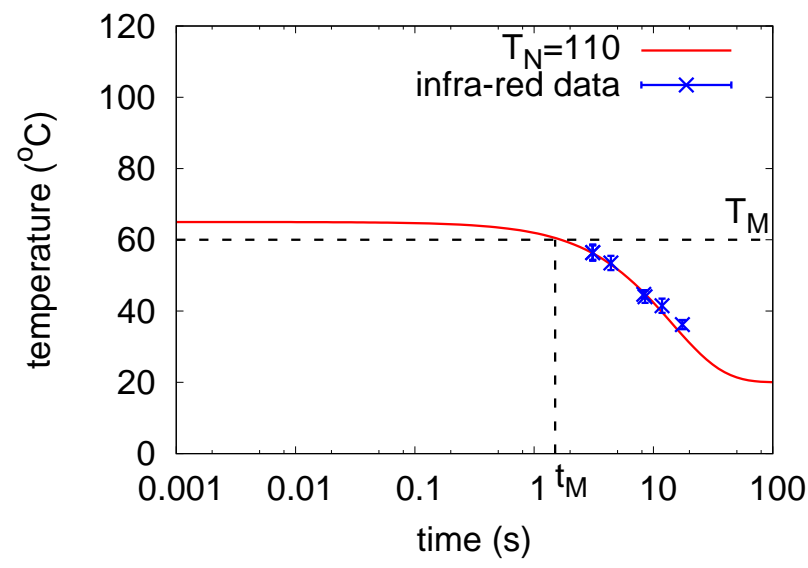

Figure 3: Temperature evolution at the surface for $T_{N}=110^{\circ} \mathrm{C}$, given by Eq. (2) compared to infra-red imaging measurements [30].

feed rate is varied $U_{0}=1.8-3 \mathrm{~mm} / \mathrm{s}$. By assuming a constant volume flux, the feed rate relates to the extrusion speed via

$$
U_{0}=U_{N}\left(\frac{R_{N}}{R_{0}}\right)^{2},
$$

where $R_{0}=0.9 \mathrm{~mm}$ is the radius of solid feedstock filament.

In particular (as in Ref. [30]), Fig. 2 shows the time taken for the top surface to reach $10 \%$ crystallinity, denoted $t_{10}$, plotted against feed rate, for three different print temperatures. For sufficiently high print temperature $\left(T_{N} \geq 110^{\circ} \mathrm{C}\right), t_{10}$ is approximately constant with increasing feed rate; the slight decrease observed in Fig. 2 is expected to be a result of increased convection at higher feed rates. Increasing the print temperature from $T_{N}=110$ to $140^{\circ} \mathrm{C}$ extends $t_{10}$, since the melt is above $T_{M}$ for longer time. On the other hand, for $T_{N}=90^{\circ} \mathrm{C}$ there is a significant decrease in $t_{10}$ with increasing print speed - a clear signature of flow-enhanced nucleation.

We propose that flow-enhanced crystallisation is absent from the experiments with print temperatures $T_{N} \geq$ $110^{\circ} \mathrm{C}$ due to stretch relaxation prior to the filament temperature reaching the melt point $T_{M}$. This hypothesis can be tested by comparing the time taken for the top surface to cool below the melt temperature $T_{M}$ to the Rouse time $\tau_{R}$ of the polymer, which governs stretch relaxation [16], as discussed in the following section.

\subsection{Surface cooling and stretch relaxation}

Here we compare surface cooling to the time taken for polymer stretch to relax to equilibrium.

Fig. 3 shows temperature at the top surface, measured using infra-red imaging [30], as a function of time for print temperature $T_{N}=110^{\circ} \mathrm{C}$. Since $t_{10} \sim 35 \mathrm{~s}$ in this case, we assume the effect of latent heat is small in the region of the infra-red measurements. (See Appendix A for further discussion on the magnitude of latent heat.) Thus, the surface temperature can be described by the exponential 
decay

$$
T_{\text {surf }}(t)=T_{\text {mid }} \exp (-A t)+T_{a},
$$

where $T_{a}$ is the ambient temperature, $T_{\text {mid }}=\left(T_{N}-T_{a}\right) / 2$, and $A=0.07 \mathrm{~s}^{-1}$ is chosen to fit the infra-red measurement. We find it takes approximately $t_{M} \sim 1 \mathrm{~s}$ for the surface to cool below $T_{M}$ for $T_{N}=110^{\circ} \mathrm{C}$, as shown in Fig. 3. A full calculation of the cross-sectional temperature profile is given later.

The Rouse time of the printing material can be extracted by fitting the linear rheology data $\left(G^{\prime}, G^{\prime \prime}\right)$ to the Likhtman \& McLeish model [33], and is executed using Reptate software [34] (see Appendix B). The fitting yields the entanglement molecular weight $M_{e}=4.8 \mathrm{~kg} / \mathrm{mol}$, the plateau modulus $G_{e}=9.2 \times 10^{5} \mathrm{~Pa}$ and the entanglement time $\tau_{e}^{0}\left(T_{0}\right)=1.9 \times 10^{-5} \mathrm{~s}$, where $T_{0}=60^{\circ} \mathrm{C}$ is the reference temperature. The Rouse time is then given by

$$
\tau_{R}(T)=\tau_{e}^{0} Z^{2} a(T),
$$

where

$$
Z=\frac{M_{w}}{M_{e}},
$$

is the entanglement number and

$$
a(T)=\exp \left(\frac{-C_{1}\left(T-T_{0}\right)}{T+C_{2}-T_{0}}\right),
$$

is the Williams-Landel-Ferry (WLF) equation [35] obtained from time-temperature superposition of the linear rheology data $\left(G^{\prime}, G^{\prime \prime}\right)$; here $C_{1}=6^{\circ} \mathrm{C}^{-1}$ and $C_{2}=123^{\circ} \mathrm{C}$.

The Rouse time increases with decreasing temperature and eventually diverges at the glass transition temperature $T_{g}=-60^{\circ} \mathrm{C}$. We can approximate the time taken for the stretch to relax by considering the Rouse time at the initial temperature $T_{\text {surf }}(0)$. For $T_{N}=110^{\circ} \mathrm{C}$, we have $T_{\text {surf }}(0)=65^{\circ} \mathrm{C}$ from Eq. (2) and a Rouse time of $\tau_{R}\left(T_{\text {surf }}(0)\right) \approx 6 \times 10^{-3} \mathrm{~s}$ from Eq. (3). Thus, $\tau_{R} \ll t_{M}$; the stretch relaxation time is much shorter than the time taken to drop below the melt temperature. Consequently, there will be no residual stretch at the onset of nucleation for this print temperature, and crystallisation will be governed by quiescent kinetics. Similarly for $T_{N}=140^{\circ} \mathrm{C}$.

\subsection{Quiescent crystallisation and saturation}

Having established that $T_{N}=110$ and $140^{\circ} \mathrm{C}$ are not affected by flow-enhanced nucleation, we move to a model to capture quiescent crystallisation kinetics. Quiescent polymer nucleation and crystallisation kinetics are described by the Schneider rate equations [37]:

$$
\begin{array}{ll}
\dot{\phi}_{3}=8 \pi \dot{N}(T), & \left(\phi_{3}=8 \pi N\right) \\
\dot{\phi}_{2}=G(T) \phi_{3}, & \left(\phi_{2}=8 \pi R_{t o t}\right) \\
\dot{\phi}_{1}=G(T) \phi_{2}, & \left(\phi_{1}=S_{t o t}\right) \\
\dot{\phi}_{0}=G(T) \phi_{1}, & \left(\phi_{0}=V_{t o t}\right)
\end{array}
$$

where $N$ is the the number of nuclei per unit volume, $R_{t o t}$ is the total radius, $S_{t o t}$ is the total surface area and $V_{t o t}$ is
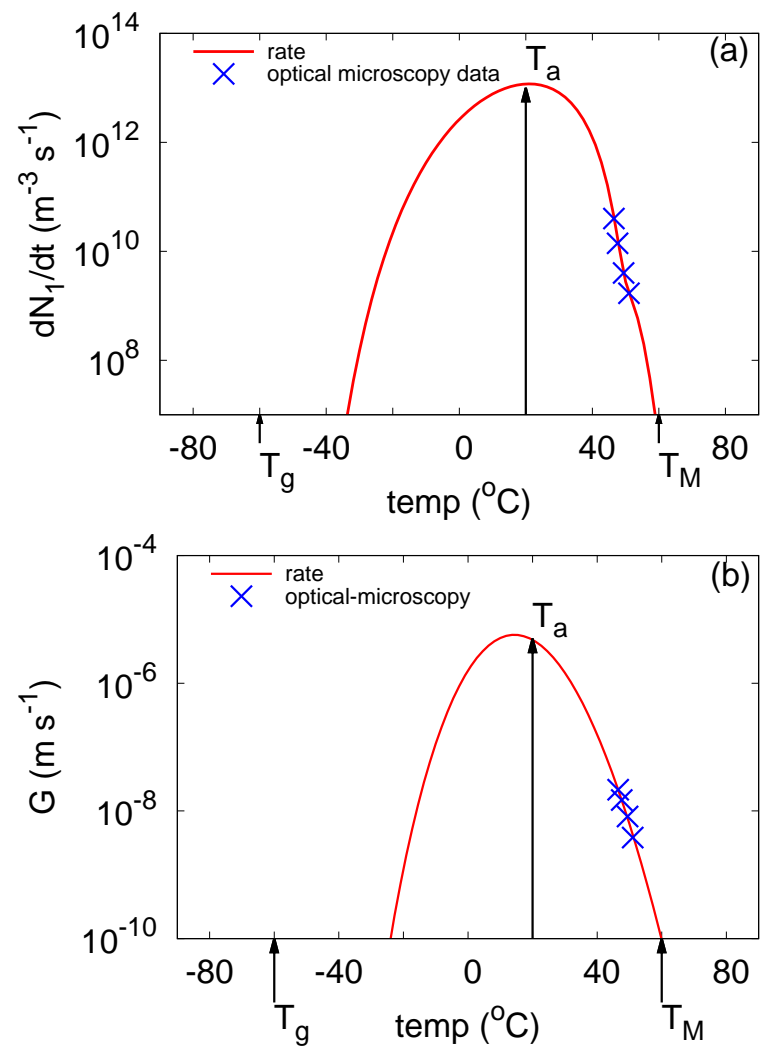

Figure 4: (a) Quiescent nucleation rate $\dot{N}_{1}$ (Eq. 10) and (b) crystal growth rate (Eq. 9) fitted to optical microscopy data for PCL from Ref. [36]. Melting temperature is $T_{M}=60^{\circ} \mathrm{C}$ and the glass transition temperature is $T_{g}=-60^{\circ} \mathrm{C}$.

the total volume of nuceli. The crystal growth rate is denoted $G$ and the nucleation rate is denoted $\dot{N}$. To correct for impingement we use the Avrami equation [38]

$$
\phi_{0}=-\ln \left(1-\xi_{g}\right)
$$

where $\xi_{g}$ is the degree of space filling, which is related to the degree of crystallinity via $\xi=V_{\max } \xi_{g}$. Here, we set $V_{\text {max }}=0.4$ (see Appendix D).

The nucleation rate that enters the Schneider rate equations is given by

$$
\dot{N}(T)=\dot{N}_{q}^{0}(T)\left(1-\frac{N_{q}}{N_{q, \max }}\right),
$$

where $\dot{N}_{q}^{0}$ is the quiescent nucleation rate and the maximum number of quiescent nuclei allowed due to saturation effects is denoted $N_{q, \max }$.

The temperature dependence of both the nucleation rate and the crystal growth rate is determined by the existence of two competing effects as described by Lauritzen \& Hoffman [42]. For PCL, the crystal growth is written as

$$
G(T)=\frac{G_{\max }}{a(T)} \exp \left(-b_{g} T^{2}\right),
$$

where $a(T)$ is given by the WLF Eq. (5). We choose parameters $G_{\max }$ and $b_{g}$ to fit the optical-microscopy mea- 
Table 1: Quiescent material properties for a typical semi-crystalline printing material polycaprolactone (PCL), obtained either directly from the literature or by fitting to experimental data available in the literature.

\begin{tabular}{|c|c|c|c|c|}
\hline PCL Property & Notation & Value & Units & Source \\
\hline \multirow[t]{2}{*}{ Molecular Weight } & $M_{w}$ & 96.7 & kg mol-1 & {$[30]$} \\
\hline & $M_{n}$ & 57.1 & $\mathrm{~kg} \mathrm{~mol}{ }^{-1}$ & {$[30]$} \\
\hline Density & $\rho$ & 1070 & $\mathrm{~kg} \mathrm{~m}^{-3}$ & {$[39]$} \\
\hline Melting Temperature & $T_{M}$ & 60 & ${ }^{\circ} \mathrm{C}$ & {$[39]$} \\
\hline Glass-Transition Temperature & $T_{g}$ & -60 & ${ }^{\circ} \mathrm{C}$ & {$[39]$} \\
\hline Entanglement Molecular Weight & $M_{e}$ & 4.8 & $\mathrm{k} \mathrm{mol}^{-1}$ & Reptate [34] with linear rheology (Appendix B) \\
\hline Plateau Modulus & $G_{e}$ & $9.2 \times 10^{5}$ & $\mathrm{~Pa}$ & Reptate [34] with linear rheology (Appendix B) \\
\hline Entanglement Time (at $\left.T_{0}\right)$ & $\tau_{e}^{0}$ & $1.9 \times 10^{-5}$ & $\mathrm{~s}$ & Reptate [34] with linear rheology (Appendix B) \\
\hline Reference Temperature & $T_{0}$ & 60 & ${ }^{\circ} \mathrm{C}$ & time-temperature superposition (Appendix B) \\
\hline WLF parameter & $C_{1}$ & 6 & ${ }^{\circ} \mathrm{C}^{-1}$ & time-temperature superposition (Appendix B) \\
\hline WLF parameter & $C_{2}$ & 123 & ${ }^{\circ} \mathrm{C}$ & time-temperature superposition (Appendix B) \\
\hline Ambient Temperature & $T_{a}$ & 20 & ${ }^{\circ} \mathrm{C}$ & {$[30]$} \\
\hline Cooling rate & $A$ & 0.07 & $\mathrm{~s}^{-1}$ & Eq. 2 fit to infra-red measurements, $[30]$ \\
\hline Crystal growth rate parameters & $\begin{array}{c}G_{\max } \\
b_{g}\end{array}$ & $\begin{array}{c}4.78 \times 10^{-4} \\
0.0043\end{array}$ & $\begin{array}{l}\mathrm{m} / \mathrm{s} \\
{ }^{\circ} \mathrm{C}^{-2}\end{array}$ & $\begin{array}{l}\text { Eq. } 9 \text { fit to data, }[36] \\
\text { Eq. } 9 \text { fit to data, }[36]\end{array}$ \\
\hline \multirow[t]{6}{*}{ Nucleation rate parameters } & $a$ & -12.28 & $m^{-3}$ & Eq. 12 fit to data, $[36]$ \\
\hline & $b$ & 639 & $\mathrm{~m}^{-3}{ }^{\circ} \mathrm{C}^{-1}$ & Eq. 12 fit to data, [36] \\
\hline & $n_{1}$ & $1.8 \times 10^{16}$ & $\mathrm{~m}^{-3} \mathrm{~s}^{-1}$ & Eq. 11 fit to data, $[36]$ \\
\hline & $n_{2}$ & $7.6 \times 10^{16}$ & $\mathrm{~m}^{-3} \mathrm{~s}^{-1}$ & Eq. 11 fit to data, [36] \\
\hline & $b_{n 1}$ & 159 & ${ }^{\circ} \mathrm{C}^{-2}$ & Eq. 11 fit to data, [36] \\
\hline & $b_{n 2}$ & 194 & ${ }^{\circ} \mathrm{C}^{-2}$ & Eq. 11 fit to data, $[36]$ \\
\hline Saturation limit & $N_{q, \max }$ & $10^{12}$ & $\mathrm{~m}^{-3}$ & Eq. 6 fit to quiescent data [30] (Fig. 5) \\
\hline Specific Heat Capacity & $C_{p}$ & 194 & $\mathrm{~J} \mathrm{~kg}^{-1{ }^{\circ} \mathrm{C}^{-1}}$ & {$[40]$} \\
\hline Heat of fusion & $H_{f}$ & 65000 & $\mathrm{~J} \mathrm{~kg}^{-1}$ & {$[40]$} \\
\hline Thermal Diffusivity (at $T_{M}$ ) & $\alpha$ & $7 \times 10^{-7}$ & $\mathrm{~m}^{2} \mathrm{~s}^{-1}$ & {$[41]$} \\
\hline
\end{tabular}

surements of growth rate by Chynoweth et al. [36] (see Table 1).

Optical microscopy is also used in Ref. [36] to extract a quiescent nucleation rate for PCL; the nucleation rate $N_{q}^{0}$ is found to be two-stage such that

$$
\dot{N}_{q}^{0}(T)=\left\{\begin{array}{l}
\dot{N}_{1}, \text { if } N<N_{c}, \\
\dot{N}_{2}, \text { if } N \geq N_{c},
\end{array}\right.
$$

with

$$
\dot{N}_{i}(T)=\frac{n_{i}}{a(T)} \exp \left(\frac{b_{n i}}{T-T_{M}}\right), \quad i=1,2,
$$

where $n_{i}$ and $b_{n i}$ are chosen to fit the data in Ref. [36] (see Table 1) (note that since the nucleation rate units are not specified correctly in Ref. [36], an assumption based on replicating these results is discussed in Appendix C). The critical number of nuclei at which the nucleation rate changes, denoted $N_{c}$, depends linearly on temperature such that

$$
N_{c}=a+b T,
$$

where $a$ and $b$ are chosen to fit the data in Ref. [36] (see Table 1).

Plots of the crystal growth rate (Eq. (9)) and nucleation rate (Eq. (11)) alongside the optical-microscopy measurements from Ref. [36] are shown in Fig. 4.
We solve the Schneider rate Eqs. (6), with Eqs. (9) and (11) for the nucleation and growth rates, alongside the typical cooling profile shown in Fig. 3 for $T_{N}=110^{\circ} \mathrm{C}$. The corresponding nucleation and crystallisation kinetics are shown in Fig. 5. In particular, nucleation occurs as soon as the temperature drops below the melt point, whereas the onset of crystal growth is much later, occurring at temperatures closer to $T_{a}$. Since the temperature decays to $T_{a}$ and stays above the glass transition temperature $T_{g}$, crystallisation continues until the space is filled $\xi_{g}=1$ and $\xi_{\max }=0.4$.

Under quiescent conditions, allowing the nuclei to saturate affects the total number of nuclei created $N_{t o t}$ and therefore affects crystallisation times. That is, decreasing $N_{q, \max }$ restricts $N_{t o t}$ and prolongs crystallisation. Thus, saturation limit $N_{q, \max }=10^{12}$ is chosen to fit the Raman spectroscopy measurements [30], as shown in Fig. 5 (i.e. $t_{10} \sim 35 \mathrm{~s}$ for $T_{N}=110^{\circ} \mathrm{C}$ assuming the absence of flow effects (Fig. 2)).

Since the nucleation and crystal growth rates increase exponentially with decreasing temperature, it is not experimentally feasible to measure $\dot{N}$ and $G$ directly at low temperatures. Therefore, we rely on the extrapolation of Eqs. (9) and (11) to the ambient temperature $T_{a}$ in our nonisothermal model. As shown in Appendix D, we find that this extrapolation can accurately reproduce the transient 

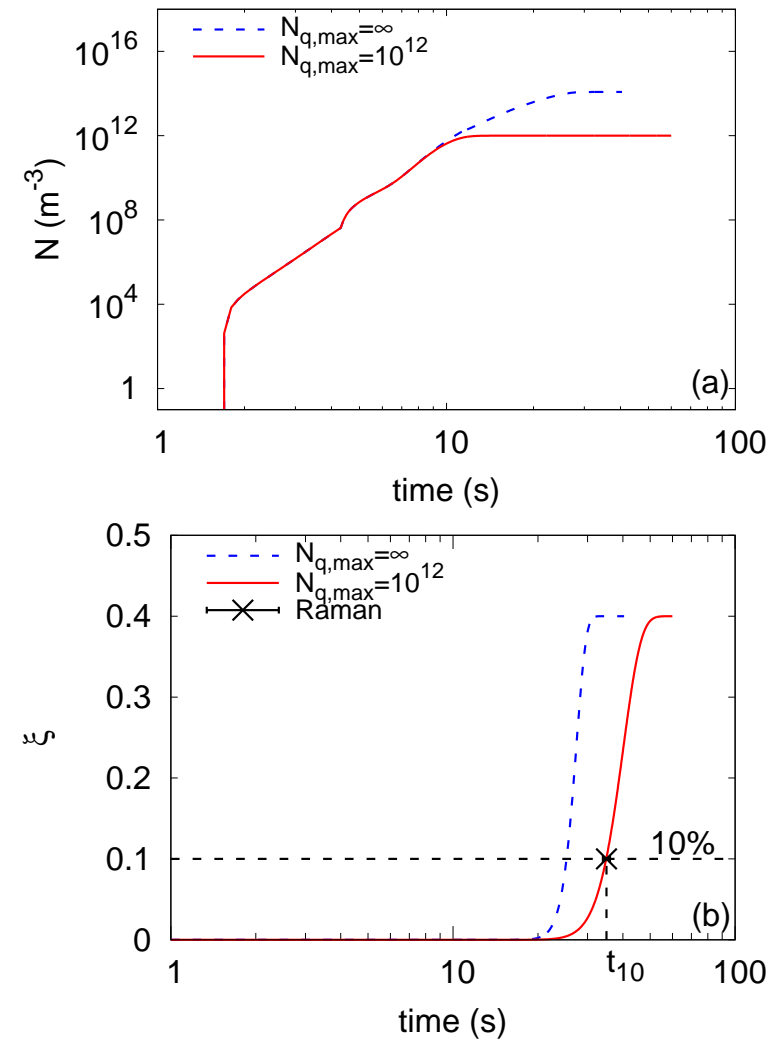

Figure 5: (a) Number of nuclei $N$ and (b) degree of crystallinity $\xi$ plotted against time corresponding to the cooling profile given by Eq. (2) and $T_{N}=110^{\circ} \mathrm{C}$ in the absence of flow. Note the separation in quiescent nucleation and crystallisation time scales for PCL. We set $N_{q, \max }=10^{12}$ to fit time to $10 \%$ crystallisation at the surface $t_{10}$ to the in-situ Raman spectroscopy data [30]. See Appendix D for further details.

crystallisation measurements from Ref. [30], at least up to $\xi=0.15$. Furthermore, the saturation limit $N_{q, \max }=10^{12}$ is consistent for two different print temperatures $T_{N}=110$ and $140^{\circ} \mathrm{C}$, which induce very different thermal histories, indicating that saturation is independent of temperature.

In this section we have determined from material characterisation of PCL the various quiescent material properties to be used in our flow-enhanced crystallisation model. These are summarised in Table 1 . These material properties give crystallisation transients that are in agreement with the in-situ Raman spectroscopy data from Ref. [30], as shown in Appendix D and Fig. 9.

\section{Flow-Enhanced Crystallisation Model}

Having established the quiescent model parameters, we now introduce a molecular flow-enhanced crystallisation model to describe the crystallisation kinetics at the lowest print temperature $T_{N}=90^{\circ} \mathrm{C}$, as shown in Fig. 2 . We require only a single extra parameter $\eta$ to model how flow effects crystallisation, as discussed in the following sections.

\subsection{Modelling FFF printing process}

During FFF a solid thermoplastic filament is fed into a heated nozzle via a pinch-roller mechanism and melted. Typical (radially-averaged) extrusion speeds of the molten material at the nozzle exit $U_{N}$ are between $10-100 \mathrm{~mm} / \mathrm{s}$ and are limited by thermal diffusivity in the nozzle; the maximum feed velocity is correlated with the print temperature $T_{N}$ in Ref. [43].

The melt is deposited onto a build plate, as the nozzle head traverses the $x y$-plane, with speed $U_{L}$. Upon deposition, the polymers deform as the flow direction changes from the vertical to the horizontal (90 degrees). The thickness of the deposit is restricted by the gap between the nozzle and the build plate $H$; the cross-sectional area and shape is determined by the balance of speeds $U_{N}$ and $U_{L}$ in order to conserve mass (prevent over/under extrusion), as well as rheological effects such as die swell and shrinkage [44, 45]. Recently, Comminal et al. [46] and Gleadall et al. [13] have developed models to predict the deposit shape, although non-Newtonian effects are not accounted for.

The deposited melt then cools, solidifies and welds with adjoining material. This process is repeated layer-by-layer to construct a 3D object. Infra-red imaging has been used to measure the temperature evolution at the weld [47] and finite-element models have been used to simulate temperature gradients at the nozzle exit [48], as well as in deposited layers [49]. Laser-assisted heating can be used to improve weld strength [50], however without a molecular understanding of the effect of temperature on the melt dynamics, this technique cannot be used optimally.

To model the FFF process, we use the method proposed by McIlroy \& Olmsted [12], which assumes the melt reaches steady state and a uniform print temperature $T_{N}>$ $T_{M}$ within the nozzle. We assume semi-crystalline materials become fully melted (and amorphous) in the nozzle and do not consider degradation due to heating. Deposition is assumed to be sufficiently fast that there is no cooling or polymer relaxation during this time and the geometry of the deposit is predetermined, neglecting die swell and shrinkage. We then implement a prescribed axisymmetric cooling profile at the surface of the deposited filament, where the cooling rate is chosen to fit infra-red measurements.

In the following sections, we briefly describe the polymer constitutive model (Rolie-Poly) and state the governing equations that describe the three FFF stages: 1) flow through the nozzle 2) deposition flow and 3) cooling, relaxation and crystallisation. For further details of the flow model see Ref. [12], and for cooling in the absence of crystallisation see Ref. [31]. This FFF process model may be considered an ideal protocol for probing flow-enhanced nucleation, since all the deformation occurs above $T_{M}$, with nucleation and crystal growth occurring subsequently in the absence of flow. 

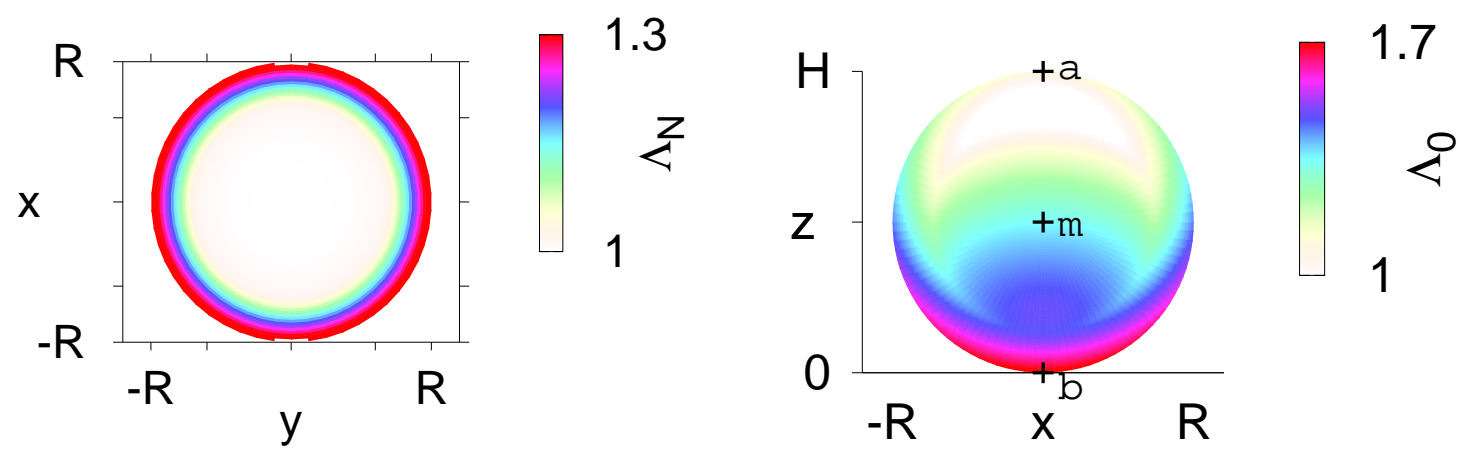

Figure 6: Stretch profiles $\Lambda=\sqrt{\operatorname{tr} \mathbf{A} / 3}$ for (a) the nozzle cross section $(\theta=0)$ and (b) the deposit cross section for $U_{N}=30 \mathrm{~mm} / \mathrm{s}$ and $T_{N}=90^{\circ} \mathrm{C}$. Locations $\mathrm{a}, \mathrm{m}$, and $\mathrm{b}$ (top, middle, and bottom of the deposit cross-section) are illustrated.

\subsection{Constitutive Model}

We use the Rolie-Poly model [51] for linear monodisperse polymers to describe the microstructural evolution of the polymer chains during flow and relaxation. Based on Doi-Edwards tube theory [16] for entangled polymer melts, where each polymer chain is assumed to be confined to a tube region, Rolie-Poly employs a single-mode constitutive equation for stress, making it less computationally expensive than the full GlaMM model [52]. The theoretical tube serves to constrain the diffusive motion of a polymer chain to reptation along the tube contour length, thus representing topological constraints, or entanglements, due to neighboring polymer chains.

The microstructure of the polymer melt is described by the conformation tensor

$$
\mathbf{A}=\frac{\langle\mathbf{R R}\rangle}{2 R_{g}^{2}}
$$

Here $\mathbf{R}$ is the end-to-end vector of the polymer chain, and the polymer's radius of gyration is denoted $R_{g}$. Tensor $\mathbf{A}$ obeys the Rolie-Poly model [51]

$$
\begin{aligned}
\frac{D \mathbf{A}}{D t} & =\left(\mathbf{K} \cdot \mathbf{A}+\mathbf{A} \cdot \mathbf{K}^{T}\right)-\frac{1}{\tau_{d}(T)}(\mathbf{A}-\mathbf{I}) \\
& -\frac{2}{\tau_{R}(T)}\left(1-\frac{1}{\Lambda}\right)\left(\mathbf{A}+\frac{\beta}{\Lambda}(\mathbf{A}-\mathbf{I})\right) .
\end{aligned}
$$

Here the Lagrangian derivative for velocity $\mathbf{u}$ is given by $\frac{D}{D t}=\frac{\partial}{\partial t}+(\mathbf{u} \cdot \nabla)$ and $\mathbf{K}=\nabla_{\alpha \beta} u_{\alpha}$ defines the velocity gradient tensor. Polymer stretch is written as

$$
\Lambda=\sqrt{\frac{\operatorname{tr} \mathbf{A}}{3}}
$$

where trA denotes the trace of tensor $\mathbf{A}$.

The first term on the right-hand side of Eq. (14) represents how the polymers stretch and orient in a flow field. Reptation and Rouse relaxation are governed by the last two terms, respectively. The degree of convective constraint release (CCR) is controlled by $\beta$.

The reptation time $\tau_{d}$ is defined by

$$
\tau_{d}(T)=3 \tau_{e}^{0} Z^{3}\left(1-\frac{3.38}{\sqrt{Z}}+\frac{4.17}{Z}-\frac{1.55}{\sqrt{Z}^{3}}\right) a(T),
$$

where $a(T)$ is the WLF Eq. (5), $Z$ is the entanglement number (Eq. (4)) and $\tau_{e}^{0}$ is the entanglement time (Table $1)$. Reptation also slows down as temperature decreases, with $\tau_{d}$ and $\tau_{R}$ eventually diverging at the glass transition temperature $T_{g}$.

\subsection{Stage 1: Flow through the nozzle}

We use the cylindrical polar coordinate system $(r, \phi, s)$, where $s$ denotes the changing flow direction such that

$$
s=\left\{\begin{array}{l}
\hat{\mathbf{e}}_{z}, \text { in the nozzle } \\
\hat{\mathbf{e}}_{y}, \text { in the deposited layer. }
\end{array}\right.
$$

Assuming an axisymmetric, steady-state flow through the nozzle, the velocity profile is given by

$$
\mathbf{u}=(0,0, w(r)),
$$

in order to obey conservation mass:

$$
\nabla \cdot \mathbf{u}=0 .
$$

Steady-state conservation of momentum is given by

$$
\frac{\partial p}{\partial s}=\frac{1}{r} \frac{\partial}{\partial r}\left(r \sigma_{r s}\right)
$$

where $p$ is pressure.

The shear stress is written as

$$
\sigma_{r s}=\mu_{s} \frac{d w}{d r}+G_{e} A_{r s},
$$

where the plateau modulus $G_{e}$ is given by

$$
G_{e}=\frac{\rho R T}{M_{e}},
$$

where $R$ is the gas constant and $T$ is temperature, and the background viscosity is given by [53]

$$
\mu_{s}=\frac{\pi^{2}}{12} \frac{G_{e} \tau_{R}}{Z} .
$$

To calculate the polymer deformation within the nozzle, Eq. (20) is solved alongside the Rolie-Poly equation 

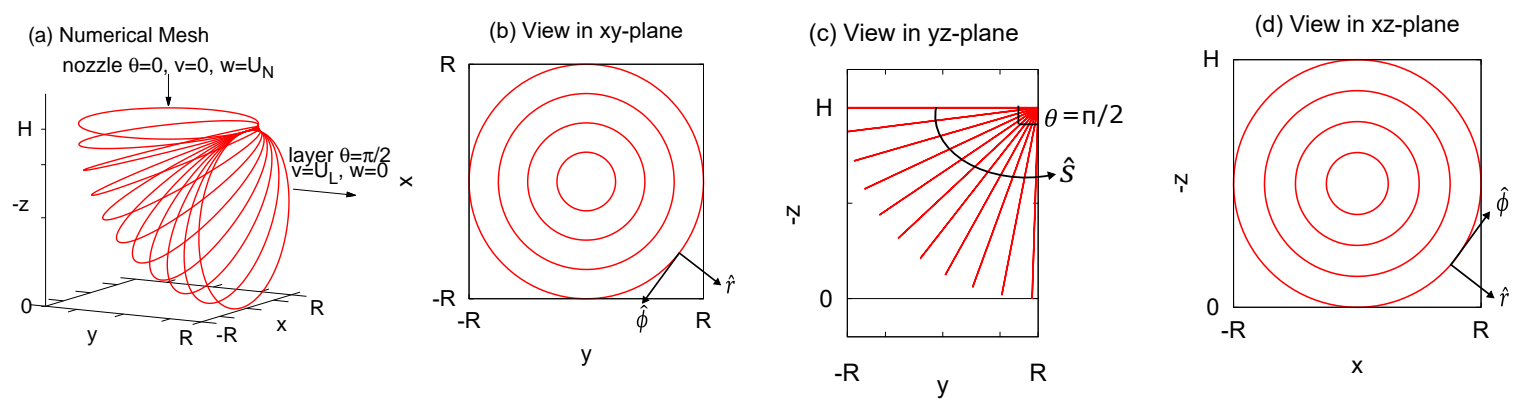

Figure 7: (a) Shape of the deposition where the outer edge of the deposit traces a smooth circular arc. Initial circular cross section $(\theta=0)$ has velocity $U_{N}$ in the $\hat{z}$-direction and final circular cross section $(\theta=\pi / 2)$ has a velocity $U_{L}=U_{N}$ in the $\hat{y}$-direction. (b) Nozzle view $(\theta=0)$ in $x y$-plane, (c) side view in the $z y$-plane and (d) layer view $(\theta=\pi / 2)$ in $x z$-plane; $r$ denotes the radial position on a plane and $\phi$ denotes the angle around a plane.

(14) with $D \mathbf{A} / D t=0$, uniform temperature $T=T_{N}$, and assuming a no-slip condition at the nozzle wall $r=R_{N}$, where $R_{N}$ is the nozzle radius. The CCR parameter $\beta$ is set to 0.5 to ensure a monotonic constitutive curve (i.e. prevent shear-banding instabilities). The pressure gradient is chosen to give a prescribed radially-averaged velocity

$$
U_{N}=\int_{0}^{R_{N}} \frac{w(r)}{\pi R_{N}^{2}} d^{2} r .
$$

A typical stretch profile for polycaprolactone rheology, $U_{N}=30 \mathrm{~mm} / \mathrm{s}$ and $T_{N}=90^{\circ} \mathrm{C}$ is shown in Fig. 6(a); the stretch is axisymmetric due to the nature of the flow and much larger near to the nozzle walls due to the high shear rate in this region. This calculation yields an initial condition for the polymer deformation to feed into the deposition flow.

\subsection{Stage 2: Deposition flow}

The shape of the deposit, shown in Fig. 7, is prescribed as follows:

1. The thickness of the deposit is $H=2 R_{N}$.

2. The width of the deposit is $W=2 R_{N}$.

3 . The outer edge of the deposit traces a smooth circular arc.

To calculate the deposition flow, the deposit is divided into a number of cross-sections (sliced from the inner nozzle exit to the outer edge of the deposit), as shown in Fig. 7. Each cross-section is defined by the angle $\theta \in[0, \pi / 2]$ between the section and the nozzle exit.

The changing flow direction is thus written as

$$
s=\sin \theta \hat{\mathbf{e}}_{y}+\cos \theta \hat{\mathbf{e}}_{z} .
$$

Once the melt exits the nozzle, the velocity profile rapidly becomes uniform across the cross section, since the flow is low Reynold's number. Thus, in Cartesian coordinates, the velocity profile of the deposition flow can be written as

$$
\mathbf{u}=(0, v(s), w(s)),
$$

since we assume no (spreading) flow in the $x$-direction.

In order to conserve mass, we require

$$
v(s(\theta))=U_{N} \frac{A(\theta)}{\pi R_{N}^{2}} \sin \theta \quad ; \quad w(s(\theta))=U_{N} \frac{A(\theta)}{\pi R_{N}^{2}} \cos \theta,
$$

where $A(\theta)$ is the area of the cross-section at angle $\theta$. Since in this case the final shape is prescribed to be circular $\left(H=W=2 R_{N}\right)$, we have $A=\pi R_{N}^{2}, \forall \theta$. The absolute cross-sectional velocity is thus given by

$$
U(s)=\sqrt{v^{2}+w^{2}}=U_{N}, \forall s,
$$

and is the same for each cross section so that $U_{L}=U(s(\pi / 2))=U_{N}$.

During deposition, the polymer deformation is simply advected with the velocity gradient tensor such that

$$
(\mathbf{u} \cdot \nabla) \mathbf{A}=\mathbf{K} \cdot \mathbf{A}+\mathbf{A} \cdot \mathbf{K}^{T},
$$

where

$$
\mathbf{K}=\left(\begin{array}{ccc}
0 & 0 & 0 \\
v_{x} & v_{y} & v_{z} \\
w_{x} & w_{y} & w_{z}
\end{array}\right)
$$

Here subscripts denote partial Cartesian derivatives in the respective directions.

Solving Eq. (29) alongside the Rolie-Poly equation (14) with uniform temperature $T=T_{N}$ for the prescribed deposition shape yields the deformation induced by the deposition flow prior to the onset of cooling, nucleation and crystallisation.

A typical cross-sectional stretch profile is shown in Fig. 6 (b) for polycaprolactone rheology, $U_{N}=30 \mathrm{~mm} / \mathrm{s}$ and $T_{N}=90^{\circ} \mathrm{C}$; the bottom half of the layer has a higher degree of stretch due to the nature of the deposition process.

\subsection{Stage 3. Cooling, relaxation and crystallisation}

Once the extruded material reaches the build plate (at $\theta=\pi / 2$ ) velocity gradients become zero (i.e. $\mathbf{K}=0$ ), and the deposit begins to cool via a combination of radiation, convection with the air and conduction with the build 
plate. However, the temperature and molecular-weight dependence of polymer heat transfer coefficients is not very well understood.

For simplicity, we assume axisymmetric cooling such that $T \equiv T(r, t)$. In light of the infra-red measurements shown in Fig. 3, which are approximately independent of speed in the range $U_{N} \in(20,40) \mathrm{mm} / \mathrm{s}$, we assume that temperature at the free surface decays exponentially with a constant cooling rate $A$. This gives the following boundary condition at the free surface

$$
T\left(R_{N}, t\right)=T_{m i d} \exp (-A t)+T_{a}
$$

and is equivalent to Eq. (2).

We then solve the one-dimensional heat equation

$$
\frac{\partial T}{\partial t}=\alpha \frac{1}{r} \frac{\partial}{\partial r}\left(r \frac{\partial T}{\partial r}\right)+\frac{H_{f}}{C_{p}} \dot{\xi}
$$

where $\alpha$ is thermal diffusivity, $H_{f}$ is latent heat of crystallisation, $C_{p}$ is the specific heat capacity and $\dot{\xi}$ is the crystallisation rate. The thermal parameters $\alpha, C_{p}$ and $H_{f}$ are assumed to be constant with temperature for simplicity and are given in Table 1.

The polymer microstructure begins to relax via

$$
\frac{d \mathbf{A}}{d t}=-\frac{1}{\tau_{d}(T)}(\mathbf{A}-\mathbf{I})-\frac{2}{\tau_{R}(T)}\left(1-\frac{1}{\Lambda}\right)\left(\mathbf{A}+\frac{\beta}{\Lambda}(\mathbf{A}-\mathbf{I})\right) .
$$

Once $T<T_{M}$, the polymer will nucleate and crystals will grow according to the Schneider rate Eqs. (6).

In the case of flow, the nucleation rate is a linear combination of quiescent nucleation and flow-enhanced nucleation such that

$$
\dot{N}=\dot{N}_{q}+\dot{N}_{f}
$$

As found via kinetic Monte-Carlo simulations [54], this nucleation rate can be written in the empirical form

$$
\dot{N}(T, \Lambda)=\underbrace{\dot{N}_{q}^{0}(T)}_{\text {quiescent }}(\overbrace{\left(1-\frac{N_{q}}{N_{q, \text { max }}}\right)}^{\text {saturation }}+\overbrace{\eta\left(\Lambda^{4}-1\right)}^{\text {stretch }}),
$$

where $\dot{N}_{q}^{0}$ is the quiescent nucleation rate (Eq. (10)) and $\eta$ is a model parameter that tunes the number of extra nuclei created by the flow; $\eta$ can be chosen to fit to experimental data. This empirical form is also used in the work of Steenbakkers \& Peters et al. (e.g. see Refs. [55, 56, 37]).

When the temperature approaches $T_{g}$, relaxation and crystallisation will be arrested due to the nature of the WLF equation (5) and the nucleation and crystal growth curves (Fig. 4).

\section{Results}

We solve Eqs. (6), (7-11) and (31-35) using a RungeKutta method to calculate the crystallisation kinetics for three print temperatures $T_{N}=140,110$ and $90^{\circ} \mathrm{C}$. We investigate the effect that increasing the feed rate $U_{0}$ has on the crystallisation kinetics.

\subsection{Transient Crystallisation}

In this section, we compare the model predictions of crystallisation to the transient Raman spectroscopy measurements described in Ref. [30]. In particular, Fig. 8 shows that the predicted time to reach $10 \%$ crystallinity is in quantitative agreement with the experimental data discussed earlier (Fig. 2) for the three print temperatures.

Note that the Raman measurement yields a depthaveraged crystallinity, since the probe penetrates approximately a quarter of the deposited filament thickness. Consequently, for comparison to the experimental data, we take the depth-averaged crystallinity

$$
\xi^{a v}(t)=\frac{4}{H} \int_{3 H / 4}^{H} \xi(z, t) d z
$$

along the centre-line $x=0$, and the corresponding average time to $10 \%$ crystallinity, $t_{10}^{a v}$.

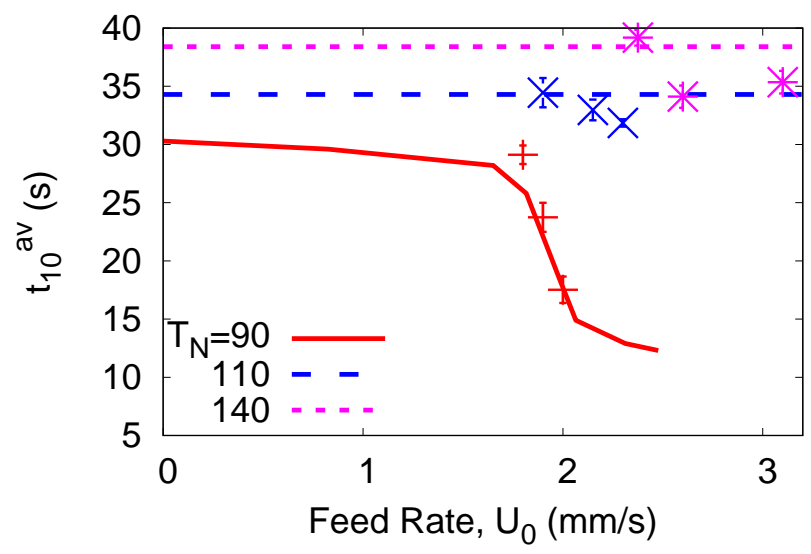

Figure 8: Depth-averaged time to $10 \%$ crystallisation $t_{10}^{a v}$ plotted against feed rate $U_{0}$ measured using Raman spectroscopy [30] (points) compared to model prediction (lines) for print speeds $T_{N}=$ 140,110 and $90^{\circ} \mathrm{C}$. Our flow-enhanced crystallisation model is in quantitative agreement with the experiments for $\eta=10^{14}$.

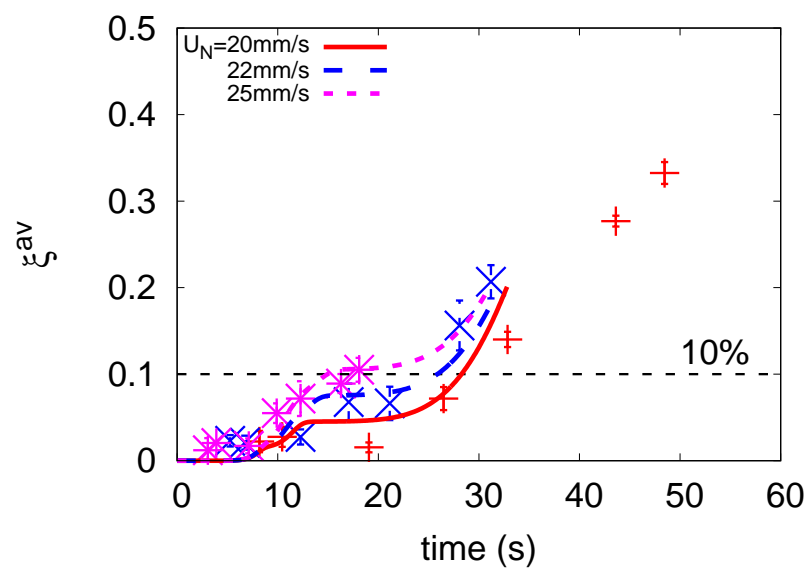

Figure 9: Evolution of the depth-averaged degree of crystallinity $\xi^{a v}$ measured using Raman spectroscopy [30] (points) and predicted by the model (lines) for $T_{N}=90^{\circ} \mathrm{C}$, where we observe flow-enhanced crystallisation. We have set $\eta=10^{14}$. 
At the two higher print temperatures, $t_{10}^{a v}$ is approximately constant with increasing feed rate and crystallisation is governed by quiescent kinetics. That is, the polymer stretch has fully relaxed to $\Lambda=1$ at $T<T_{M}$, so that nucleation is not affected by stretch and proceeds to the saturation point $N_{q, \max }$, as discussed earlier (Fig. 5). Thus, crystal growth and therefore $t_{10}^{a v}$ is independent of print speed.

It should be noted that at these high print temperatures, as well as the stretch fully relaxing prior to $T<T_{M}$, the filament also reaches a uniform temperature in this time. Since the temperature evolution is equivalent at the surface and the centre once $T<T_{M}$, nucleation occurs uniformly across the filament so that $\xi=\xi^{a v}$ everywhere on the filament cross section.

In contrast, at the lower print temperature of $T_{N}=$ $90^{\circ} \mathrm{C}$, there is a steep decrease in $t_{10}^{a v}$ at a feed rate of $U_{0} \approx 1.8 \mathrm{~mm} / \mathrm{s}$. We find quantitative agreement with the experimental data for $\eta=10^{14}$. This accelerated crystallisation is due to residual polymer stretch $(\Lambda>1)$ at $T<T_{M}$ enhancing the nucleation rate via Eq. (35) so that $N_{t o t}>N_{q, \max }$. Further details are given in Sec. 5.1.

At feed rates just beyond the highest experimental rate $\left(U_{0}>2 \mathrm{~mm} / \mathrm{s}\right)$, the model predicts a plateau in $t_{10}^{a v}$ indicating a saturation of flow effects with increasing polymer stretch (see Sec. 5.3 onwards for further discussion regarding this saturation effect).

The transient crystallisation curves for $T_{N}=90^{\circ} \mathrm{C}$, shown in Fig. 9, demonstrate very different behaviour to those shown in Appendix D for the higher quiescent print temperatures. In particular, we observe a pronounced twostage growth of $\xi^{a v}$ over time in agreement with the experimental transients. That is, there exists crystal growth followed by a plateau region, where $\xi^{a v}$ is constant in time, before crystal growth resumes.

Since the model can predict crystallisation times and the corresponding crystal morphology at any position within the deposited filament, the model is able to reveal the origin of this step in the transient data, as discussed in the following section.

\subsection{Spatial Crystallisation}

Here we employ the model to predict how nucleation varies cross-sectionally across a deposited filament. In particular, Fig. 10 shows the cross-sectional profile of the total number of nuclei $N_{\text {tot }}$ that are created during cooling. In this example the feed rate is $U_{0}=2.3 \mathrm{~mm} / \mathrm{s}$, which yields extrusion velocity $U_{N}=30 \mathrm{~mm} / \mathrm{s}$ (Eq. (1)) and gives the initial cross-sectional stretch profile shown in Fig. 6(b).

Significantly more nuclei are created at the surface of the deposited filament due to flow-enhanced nucleation. In contrast, nucleation at the centre of the filament is not affected by flow, and is governed by quiescent kinetics for which nuclei saturate at $N_{q, \max }=10^{12}$. Asymmetry of the flow-enhanced boundary layer comes from the asymmetric initial stretch profile (Fig. 6(b)).

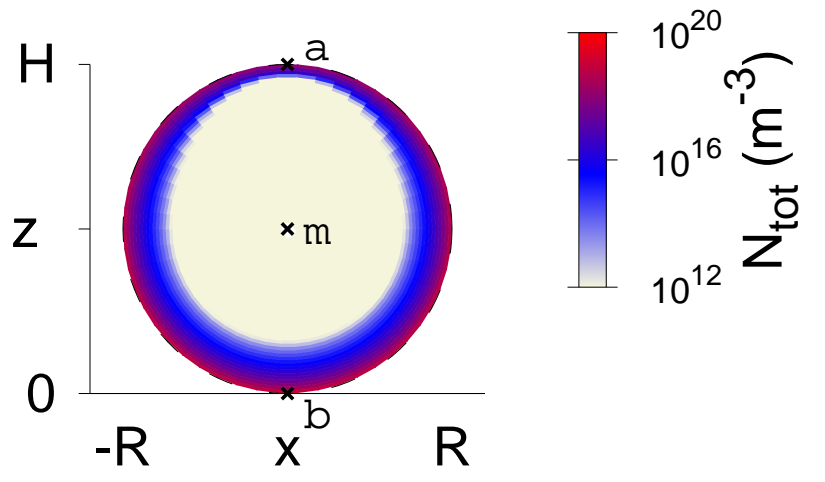

Figure 10: Cross-section profile of the total number of nuclei $N_{t o t}$ for $U_{N}=30 \mathrm{~mm} / \mathrm{s}$ and $T_{N}=90^{\circ} \mathrm{C}$. The saturation limit is $N_{q, \max }=$ $10^{12}$ and we have set $\eta=10^{14}$. Only nucleation at the surface is affected by the stretch induced by the FFF flow.

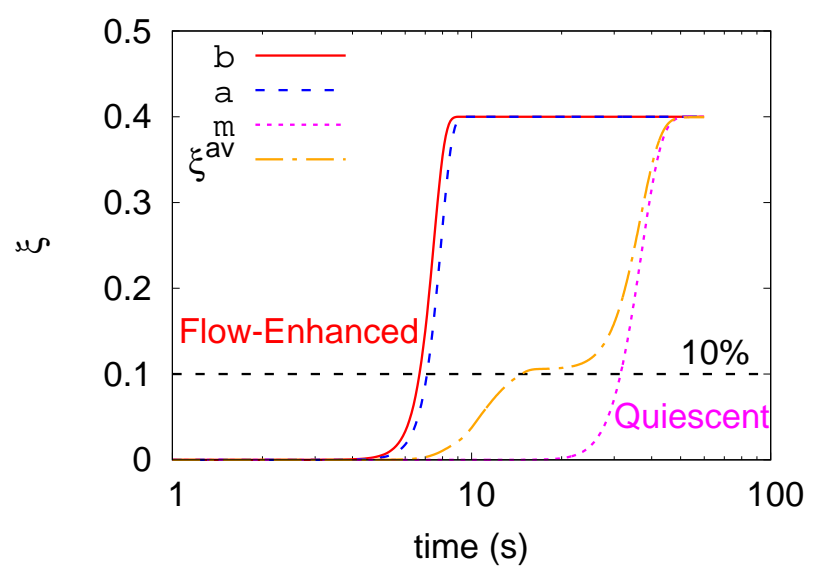

Figure 11: Evolution of the degree of crystallinity $\xi$ at the centre of the filament $\mathrm{m}$ and top/bottom free-surface locations $\mathrm{a}, \mathrm{b}$, compared to the depth-averaged crystallinity, $\xi^{a v}$ (Eq. (36)). We have set $\eta=10^{14}$.

This multi-dimensional prediction indicates how the crystal morphology varies cross-sectionally throughout deposited filament; since the number of nuclei created at the surface is orders of magnitude greater than the number created in the bulk, the surface spherulites will be significantly smaller, as determined by the Schneider rate Eqs. (6). This spatial variation in spherulite size and density is completely unapparent in the time to $10 \%$ crystallinity measurement and is only hinted at in the full transient, but can be readily seen in the modelling.

We can also consider the crystallisation kinetics at different $z$ positions on the deposit cross section; for instance, the top (apex) at $z=H$ denoted a, the middle at $z=H / 2$ denoted $\mathrm{m}$, and the bottom at $z=0$ denoted $\mathrm{b}$, as illustrated in Fig. 10. Fig. 11 shows how the degree of crystallinity evolves over time at these spatial positions.

Since the temperature remains above $T_{g}$, crystallisation proceeds to full space filling such that $\xi_{g}=1$ and $\xi_{\max }=0.4$. Thus, the degree of crystallinity ultimately becomes cross-sectionally uniform given sufficient time. The horizontal line in Fig. 11 indicates the time to reach $10 \%$ 


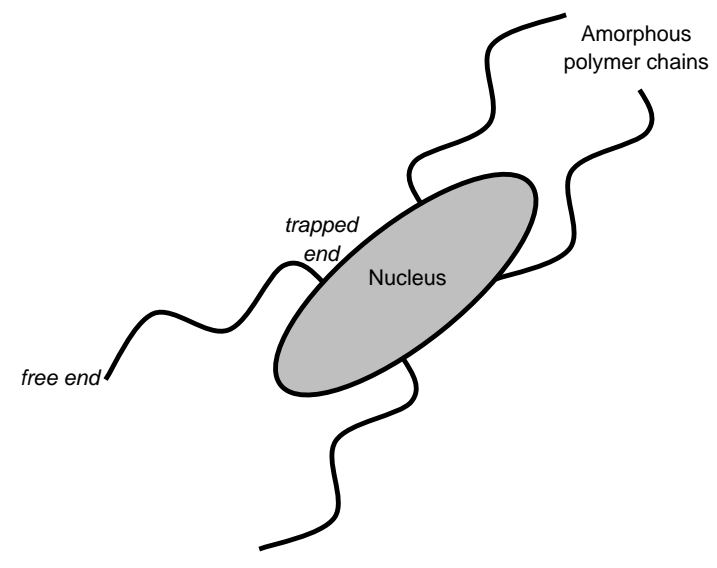

Figure 12: Nuclei trap one end of a polymer molecule forming starlike structures.

crystallinity.

There is a significant difference in the crystallisation time $t_{10}$ at the free surface positions $a, b$, compared the centre position $\mathrm{m}$. Comparing these transients to the evolution of the depth-average crystallinity $\xi^{a v}$ (Eq. (36)) reveals the origin of the step in the transient measurement; the plateau region separates earlier contributions from the flow-enhanced free-surface from the bulk contribution, which enters the calculation much later since the quiescent kinetics are significantly slower. The length of the plateau region is therefore determined by $\eta$.

During this crystal growth polymer chains become trapped at one end in the growing spherulite structures; spherulites act to cross-link the polymer chains forming a star-like architecture, as shown in Fig. 12. Consequently, the reptation time of a trapped polymer chain is reduced. Thus, it is possible for flow-enhanced crystallisation at the surface of a deposited filament to inhibit inter-diffusion between adjacent filaments, depending on $t_{10}$.

Despite acceleration due to flow, $t_{10}$ at the surface remains larger than the reptation time of the polymer; by approximating the reptation time at the coolest temperature $T_{a}$, we find that $\tau_{d} \sim t_{10} / 2$ at the surface. Thus, we expect the polymer to diffuse at least $2 R_{g}$ before becoming cross-linked by spherulites. (This inter-penetration depth is likely to be further due to non-isothermality.) Furthermore, since the nucleation density is relatively small for PCL, we expect only a proportion of chains will form star structures, whilst the remaining chains will be free to reptate in the usual way.

We consider how an increased nucleation density at the surface due to flow effects may be advantageous to the welding process in Sec. 6.

\section{Discussion}

Having established our model's ability to quantitatively describe the flow-enhanced crystallisation measurements given in Ref. [30], we now detail specifically how the poly- mer stretch induced by the FFF flow results in an inhomogeneous spherulites-size distribution and a reduction in crystallisation time at the surface. This modelling is able to explain the saturation of $t_{10}$ predicted by the model at high feed rates (Fig. 8)

\subsection{Polymer stretch and crystallisation}

Here we discuss how the relaxing polymer stretch and cooling temperature profile interact to give the flow-enhanced boundary layer shown in the previous section. We choose typical print speed $U_{N}=30 \mathrm{~mm} / \mathrm{s}$, print temperature $T_{N}=90^{\circ} \mathrm{C}$, model parameter $\eta=10^{9}$, and consider the dynamics at $\mathrm{a}, \mathrm{b}$ and $\mathrm{c}$.

Fig. 13 shows the typical cooling profile for $T_{N}=$ $90^{\circ} \mathrm{C}$. The surface locations at a and $\mathrm{b}$ have equivalent cooling profiles and immediately drop to $\sim T_{\text {surf }}(0)=$ $\left(T_{N}+T_{a}\right) / 2$ due to the imposed boundary condition. It takes $\sim t_{M}=0.003 \mathrm{~s}$ for the surface to cool below the melting temperature $T_{M}=60^{\circ} \mathrm{C}$. On the other hand, the middle of the deposit at $\mathrm{m}$ takes much longer $\left(\sim t_{M}=1 \mathrm{~s}\right)$ to reach $T_{M}$. Latent heat effects are small (see Appendix A).

Fig. 14(a) shows the stretch relaxation curve at positions $\mathrm{a}, \mathrm{b}$ and $\mathrm{m}$ for $T_{N}=90^{\circ} \mathrm{C}$. Stretch is larger at the surface positions $\mathrm{a}, \mathrm{b}$ than the middle $\mathrm{m}$ of the deposit due to the shear flow in the nozzle, and larger at the bottom $\mathrm{b}$ of the deposit than the top a due to the deposition flow. At early times the Rouse time is shorter at $m$ than at the surface $a, b$ due to the higher temperature, therefore the stretch relaxes slightly faster at the center of the deposit. The stretch becomes fully relaxed everywhere after $\sim t=0.05 \mathrm{~s}$.

Fig. 14(b,c) shows the corresponding nucleation and crystallisation curves. Flow does not effect nucleation at the centre $\mathrm{m}$ since the stretch here relaxes before the temperature cools to $T_{M}$. Thus, we observe only quiescent kinetics, with nuclei saturating at $N_{q, \max }$.

On the other hand, the stretch does increase the nucleation rate at surface positions $a, b$. Since $a, b$ have equivalent temperature history, the difference in the nucleation curves is solely a result of the stretch evolution. In particular, the total number of nuclei $N_{\text {tot }}$ created at b is greater than that at a due to a larger initial degree of stretch $\Lambda_{0}$ at the bottom surface. Since $\dot{N}_{f}=0$ and $N>N_{q, \max }$ once $\Lambda \rightarrow 1$, nucleation ceases once the stretch has relaxed, which occurs on the order of the Rouse time.

Finally, Fig. 14(c) shows the evolution of crystallinity with time for this case. Again crystallisation proceeds to full space filling since $T>T_{g}, \forall t$. The time to $10 \%$ crystallisation $t_{10}$ is indicated by the horizontal line at $\xi=0.1$. As expected, $t_{10}$ is significantly reduced at surface positions $\mathrm{a}, \mathrm{b}$ compared to the middle at $\mathrm{m}$ due to flow-enhanced nucleation.

Comparing $\mathrm{a}$ to $\mathrm{b}$ eliminates the effects of temperature, enabling a direct comparison of crystallisation kinetics arising only from different degrees of polymer stretch. 


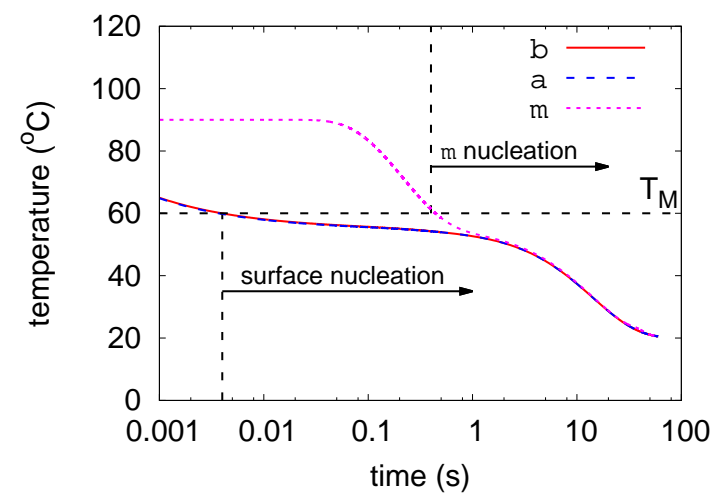

Figure 13: (a) Temperature evolution at locations a, m and b (top, middle and bottom of deposit cross-section, respectively) for $T_{N}=$ $90^{\circ} \mathrm{C}$. Surface locations a and $\mathrm{b}$ have the same cooling profile in this model. Nucleation occurs once the temperature has cooled below the melting point, $T_{M}$.
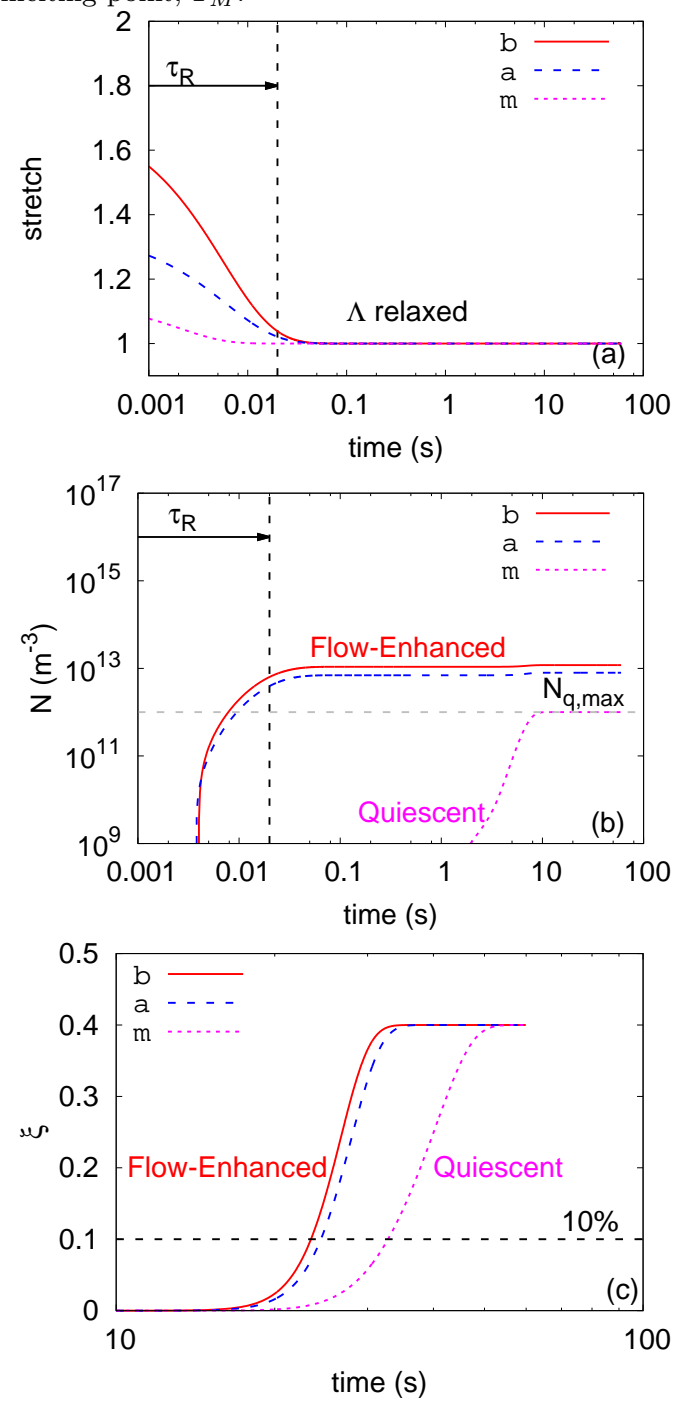

Figure 14: (a) Degree of stretch $\Lambda$, (b) number of nuclei $N$, and (c) degree of crystallinity $\xi$ plotted against time, at the surface a, b and the centre $\mathrm{m}$ of the deposit, for print temperature $T_{N}=90^{\circ} \mathrm{C}$ and print speed $U_{N}=30 \mathrm{~mm} / \mathrm{s}$. Nucleation is enhanced at the surface $\mathrm{a}, \mathrm{b}$, whereas the middle $\mathrm{m}$ follows quiescent kinetics. For corresponding temperature evolution see Fig. 13. The saturation limit is $N_{q, \max }=10^{12}$ and $\eta=10^{9}$.
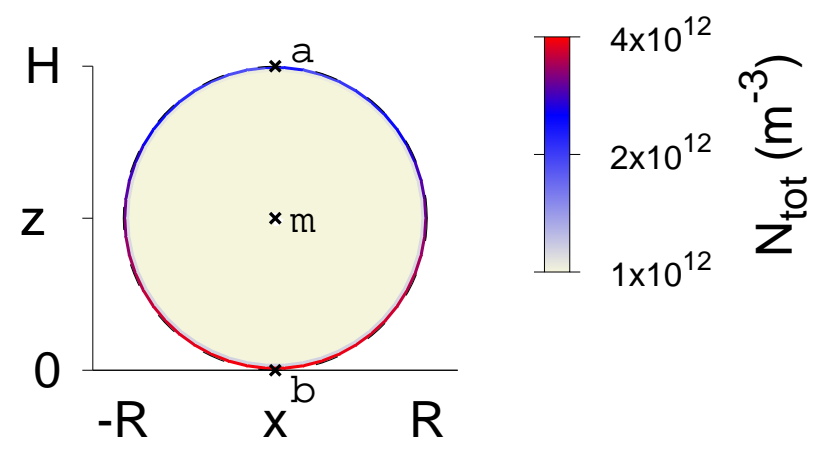

Figure 15: Cross-section profile of the total number of nuclei $N$ for $U_{N}=30 \mathrm{~mm} / \mathrm{s}, T_{N}=90^{\circ} \mathrm{C}$ and $\alpha \equiv \alpha\left(T_{a}\right)$. The saturation limit is $N_{q, \max }=10^{12}$ and $\eta=10^{9}$. Thermal diffusivity has a significant effect on the thickness of the flow-enhanced boundary layer.

Although $\Lambda_{0}$ and consequently $N_{\text {tot }}$ is much larger at b compared to a (see Figs. 14 (a) and (b)), Fig. 14(c) demonstrates that the difference in $t_{10}$ at $\mathrm{b}$ and $\mathrm{a}$ is less than a second. This counter-intuitive outcome is due to the same saturation effect seen at higher feed rates in Fig. 8, and is discussed from Sec. 5.3 onwards.

\subsection{Thermal diffusivity and Flow-Enhanced Boundary Layer}

The flow-enhanced boundary layer shown earlier in Fig. 10 illustrates regions for which $T<T_{M}$ before the polymer stretch relaxes. Thus, the thickness of this boundary is determined by the magnitude of thermal diffusivity, $\alpha$ i.e. how fast the cooling boundary penetrates the bulk of the deposited filament.

Note that thermal diffusivity is in fact temperaturedependent, and increases by nearly an order of magnitude between room temperature and the melt point [41]. Since nucleation is fast, we chose to keep diffusivity constant such that $\alpha \equiv \alpha\left(T_{M}\right)$.

On the other hand, for $\alpha \equiv \alpha\left(T_{a}\right)$, Fig. 15 shows that in this case only a very thin boundary layer of the material near the surface is affected by flow-enhanced nucleation. Furthermore, the thickness of this boundary is insufficient to contribute to the average crystallinity $\xi^{a v}$ i.e. the average calculation (Eq. 36) is dominated by quiescent crystallisation occurring in the bulk so that $\xi^{a v}$ is equivalent to $\xi$ at $\mathrm{m}$.

\subsection{Surface Crystallisation Time}

Next we employ the model to investigate why flowenhanced crystallisation appears to saturate at high feed rates i.e. the reason for plateau in $t_{10}^{a v}$ shown in Fig. 8, as well as the small difference in $t_{10}$ at the top and bottom surfaces in Fig. 14(c). We isolate the effect of stretch by only considering crystallisation at free surface positions a, b, which have equivalent temperature histories.

Fig. 16(a) shows the surface crystallisation time $t_{10}$ plotted against the total number of nuclei $N_{\text {tot }}$ and Fig. 16(b) shows $t_{10}$ plotted against the initial stretch $\Lambda_{0}$. Extrusion speed ranges from $U_{N}=1-100 \mathrm{~mm} / \mathrm{s}$; two points 

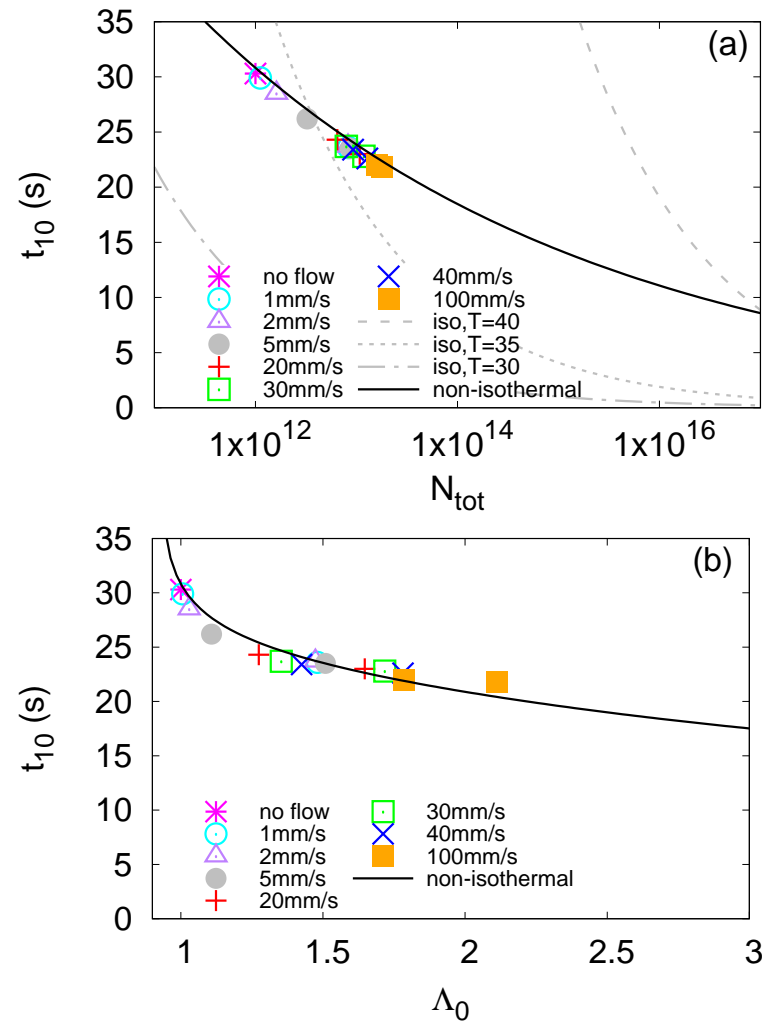

Figure 16: Time to $10 \%$ crystallisation $t_{10}$ at the surface plotted against (a) the total number of nuclei $N_{\text {tot }}$ and (b) the initial stretch $\Lambda_{0}$ for $T_{N}=90^{\circ} \mathrm{C}$ and print speeds ranging $U_{N}=1-100 \mathrm{~mm} / \mathrm{s}$. The two points for each speed represent surface positions a,b. The cooling profile for each case is shown in Fig. 13. The saturation limit is $N_{q, \max }=10^{12}$ and $\eta=10^{9}$. Lines correspond to an analytic solution for isothermal conditions (Eq. (40)) and an approximate power law (Eq. (41)) for non-isothermal conditions.

are plotted for each print speed corresponding to surface positions $\mathrm{a}$ and $\mathrm{b}\left(\Lambda_{0}\right.$ is always greater at $\left.\mathrm{b}\right)$. The case excluding flow effects $\left(\Lambda_{0}=1\right)$ is also plotted for reference; the kinetics in this case are determined by $N_{q, \max }$.

We find that only very small values of the initial stretch are needed to have a dramatic effect on $t_{10}$. Although increasing the print speed from $U_{N}=1$ to $5 \mathrm{~mm} / \mathrm{s}$ induces only small amounts of stretch $\left(\Lambda_{0}<1.1\right)$ at the top surface a, $t_{10}$ is reduced up to 10 seconds for this value of $\eta$. We then observe a saturation of these flow effects, where $t_{10}$ plateaus around $\Lambda_{0} \approx 1.1$, with $t_{10} \approx 20 \forall 1.1<\Lambda_{0}<3$. Thus, there is little change in $t_{10}$ for the range of $\Lambda_{0}$ corresponding to typical printing speeds $U_{N} \in(20,40) \mathrm{mm} / \mathrm{s}$.

This saturation of flow effects on $t_{10}$ at such a small degree of stretch is an unexpected feature of the model. To explore this behaviour further we take an analytical approach in the next section.

\subsection{Isothermal Analytic Solution}

Analytical solutions are valuable tools for identifying the underlying physics leading to counter-intuitive features in both experimental data and numerical models. In our case, we could only derive an analytical solution for $t_{10}$ for isothermal conditions. Nevertheless, this leads to interesting insights into how non-isothermality affects flowenhanced crystallisation, as discussed henceforth.

Due to the separation of nucleation and crystallisation time scales for PCL, nucleation is complete at the onset of crystallisation. Thus, we have

$$
\dot{\phi}_{3}=8 \pi \dot{N}=0 \Rightarrow \phi_{3}=8 \pi N_{t o t} .
$$

Substituting this into the subsequent Schneider rate equations (Eq. 6) and integrating three times yields

$$
\dot{\phi}_{0}=8 \pi N_{t o t} G^{3} \iiint d t^{3} \Rightarrow \phi_{0}=\frac{4 \pi}{3} N_{t o t} G^{3} t^{3},
$$

since the crystal growth rate $G \equiv G(T)$ is constant. At $10 \%$ crystallisation we have

$$
\phi_{0}=-\ln \left(1-2 \xi_{10}\right),
$$

with $\xi_{10}=0.1$. Thus,

$$
t_{10}=\left(-\frac{3 \ln \left(1-2 \xi_{10}\right)}{4 \pi N_{t o t} G^{3}}\right)^{1 / 3} .
$$

This solution is plotted as dashed line in Fig. 16(a). Reducing the temperature affects the crystal growth $G$ in Eq. (40) and shifts the curves to the right; far fewer nuclei are required to achieve the same value of $t_{10}$. (See Appendix E for Eq. (40) compared to full numerical solution of isothermal conditions.) We discuss how Eq. (40) compares with the non-isothermal case in the following section.

\subsection{Non-Isothermal Power-Law Behaviour}

Whereas isothermality yields $t_{10} \sim N_{\text {tot }}^{-1 / 3}$, as derived in the previous section, for non-isothermal conditions the dependence of $t_{10}$ on the total number of nuclei created is weaker, with a relationship closer to

$$
t_{10} \sim N_{t o t}^{-1 / 9}
$$

as plotted in Fig. 16(a).

Thus, flow-enhanced crystal growth at the surface of an FFF-deposited filament demonstrates a power-law dependence on the total number of nuclei $N_{t o t}$, where there exists a steep decrease in $t_{10}$ with increasing $N_{\text {tot }}$ followed by a slow decay to zero, as shown in Fig. 17 for a wide range of $N_{t o t}$. Since the crystallisation time is less sensitive to nucleation density for these typical non-isothermal conditions, the drop in $t_{10}$ occurs at relatively small nucleation densities so that the PCL kinetics lie in a regime where additional nuclei have little effect on the time to $10 \%$ crystallinity.

This asymptotic behaviour that forces crystallisation times to saturate for increasingly large numbers of nuclei, also manifests itself when plotting $t_{10}$ against polymer stretch (Fig. 16(b)), and leads to the unexpected plateau in $t_{10}$ at small values of $\Lambda_{0}$. We have demonstrated that 


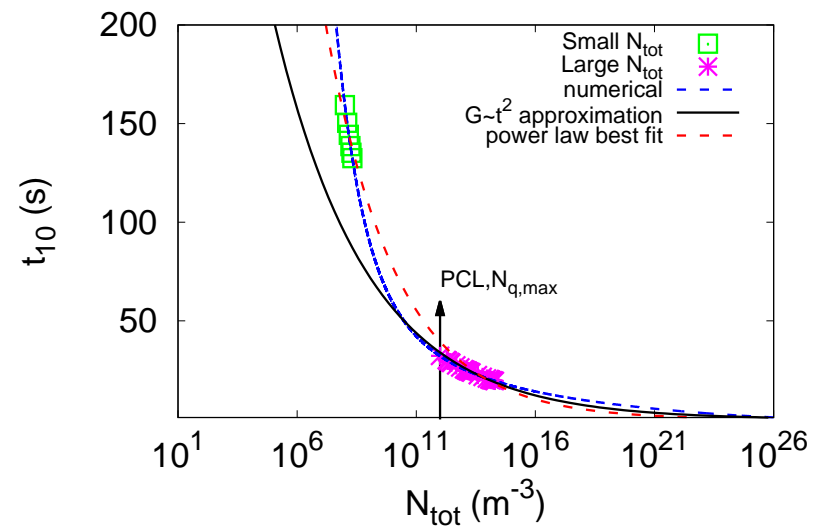

Figure 17: Time to $10 \%$ crystallisation $t_{10}$ at the surface plotted against the total number of nuclei $N_{t o t}$ for large $N_{t o t}$ regime described in Sec. 5.1 and small $N_{t o t}$ regime with $N_{q, \max }=10^{6}$ and $\eta=10^{3}$. Lines correspond to the numerical solution to Eq. (42), the approximate power law (Eq. (41)) derived by approximating $G \sim t^{2}$, and the power law of best fit.

this saturation effect with increasing polymer stretch is amplified by the cooling temperature profile present for typical FFF conditions in comparison with isothermal conditions.

The power law behaviour in Eq. (41) derives from the solution to the Schneider rate equations:

$$
\frac{\phi_{0}}{8 \pi N_{t o t}}=\int_{0}^{t_{10}} G\left(t^{\prime \prime}\right)\left[\int_{0}^{t^{\prime \prime}} G\left(t^{\prime}\right)\left[\int_{0}^{t^{\prime}} G(t) d t\right] d t^{\prime}\right] d t^{\prime \prime},
$$

where $\phi_{0}=-\ln \left(1-2 \xi_{10}\right)$ at $10 \%$ crystallisation. Due to the time-dependent nature of $G(T(t))$, an analytic solution to Eq. (42) is not possible for non-isothermal conditions. However, the $-1 / 9$ power law can be obtained by assuming the growth rate increases quadratically with time between $T \in\left(T_{N}, T_{a}\right)$ i.e. by approximating $G \sim t^{2}$. This approximate solution is plotted in Fig. 17, alongside the full numerical solution to Eq. (42), as well as the power law of best fit $t_{10} \sim N_{t o t}^{-0.147}$.

Moreover, $N_{\text {tot }}$ can be written as a function of the initial stretch $\Lambda_{0}$ as follows. Since nucleation is dominated by $\dot{N}_{f}$ and occurs whilst $\xi \ll 1$, we can write

$$
\dot{N}=\dot{N}_{q}^{0} \eta\left(\Lambda(t)^{4}-1\right) \text {. }
$$

Since the temperature is approximately constant during flow-enhanced nucleation at the surface (see Fig. 13). $\dot{N}_{q}^{0} \equiv \dot{N}_{q}^{0}(T)$ is constant. Approximately, the stretch decays according

$$
\Lambda(t)=\left(\Lambda_{0}-1\right) \exp \left(-\frac{t}{\tau_{R}}\right)+1
$$

where $\tau_{R} \equiv \tau_{R}(T)$ is also constant during nucleation, and $\Lambda_{0}$ is the initial stretch induced by the flow (Fig. 6(b)).
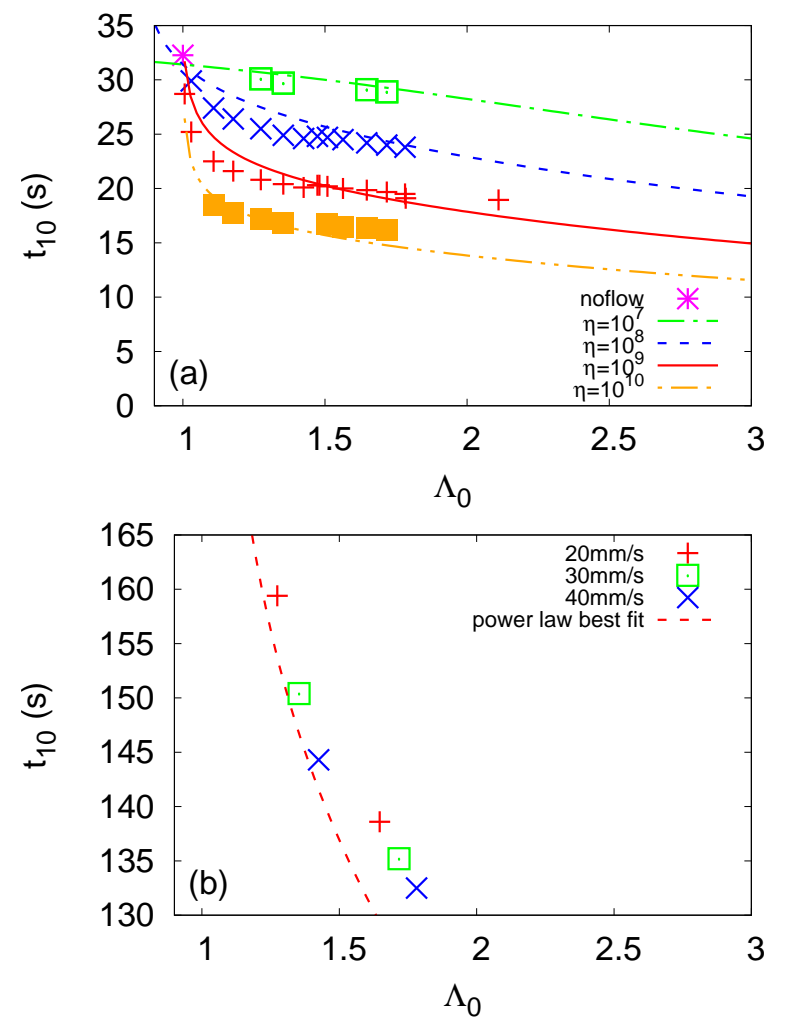

Figure 18: Time to $10 \%$ crystallisation $t_{10}$ at the surface plotted against initial stretch $\Lambda_{0}$. (a) Large $N_{\text {tot }}$ regime, described in Sec. 5.1 for various values of $\eta$. (b) Small $N_{\text {tot }}$ regime, with $N_{q, \max }=$ $10^{6}$ and $\eta=10^{3}$, for print speeds $U_{N}=20-40 \mathrm{~mm} / \mathrm{s}$. The two points for each speed represent surface positions a, b. Lines represent substituting Eq. (45) into the best-fit power law $t_{10} \sim N_{\text {tot }}^{-0.147}$ shown in Fig. 17.

Thus, we can solve Eq. (43) to give

$$
\begin{aligned}
& N_{\text {tot }}=\dot{N}_{q}^{0} \eta \tau_{R} \\
& \times\left[\left(\Lambda_{M}-1\right)^{4}+\frac{4}{3}\left(\Lambda_{M}-1\right)^{3}+2\left(\Lambda_{M}-1\right)^{2}+4\left(\Lambda_{M}-1\right)\right],
\end{aligned}
$$

where $\Lambda_{M}$ is the degree of stretch when $T(t)=T_{M}$.

Consequently, the crystallisation time $t_{10}$ can be written as a function of $\Lambda_{0}$ by substituting Eq. (45) into the approximate power-law behaviour given by Eq. (41). This theory is plotted in Fig. 16(b) and is in agreement with the numerical model predictions. (See Appendix E for Eq. (45) compared to the full numerical solution.)

\subsection{Saturation with Stretch}

For FFF-printed PCL, we have seen saturation of $t_{10}$ at small degrees of stretch, corresponding to relatively slow print speeds. Thus, a single $t_{10}$ measurement may not reveal the full effect that typical printing flow has on the cross-sectional crystal morphology.

For example, $t_{10}$ may remain approximately constant with increasing shear rate, even for very small rates; only comparison to the equivalent quiescent kinetics will reveal 
any reduction in $t_{10}$. Furthermore, despite an approximately constant $t_{10}$ in this large $N_{\text {tot }}$ regime, the increase in the number of nuclei with shear rate will lead to the growth of much smaller spherulite structures at the surface of filaments deposited at faster feed rates.

We can predict whether this saturation effect will occur by considering the total number of nuclei that are created. That is, there may or may not be a saturation of flow effects with increasing polymer stretch (or shear rate or print speed) depending on if the kinetics lie in either

1. Large $N_{\text {tot }}$ regime, where there is a slow decay in $t_{10}$ with increasing nuclei, or

2. Small $N_{\text {tot }}$ regime, where there is a steep decrease in $t_{10}$ with increasing nuclei.

The PCL kinetics we have discussed lie in the large $N_{t o t}$ regime, as determined by the saturation limit $N_{q, \max }$ (Fig. 17).

Flow-enhanced crystallisation in the large $N_{\text {tot }}$ regime always demonstrates saturation at small values of $\Lambda_{0}$. In particular, Fig. 18(a) shows how $t_{10}$ becomes approximately constant in the region $1.1<\Lambda_{0}<3$ for different values $\eta$; decreasing $\eta$ whilst ensuring $N_{f}>N_{q, \max }$ reduces the effect of flow-enhanced nucleation until only quiescent kinetics prevail, whilst increasing $\eta$ yields more flowinduced nuclei and therefore reduces the absolute value of $t_{10}$.

Thus, the large $N_{t o t}$ regime is considered beneficial for welding, in particular by ensuring inter-diffusion precedes crystal growth; even at the fastest print speeds, the extra nuclei created by the increased polymer stretch will not greatly affect $t_{10}$. Thus, inter-diffusion at the weld line will not be limited by the growth of spherulites, as discussed earlier. In Sec. 6 we discuss further advantages for an increased nucleation density near the surface of a deposited filament.

On the other hand, the small $N_{\text {tot }}$ regime is achieved in our model by reducing the saturation limit $N_{q, \max }$ and parameter $\eta$. This gives much longer crystallisation times, since $t_{10} \rightarrow \infty$ as $N_{\text {tot }} \rightarrow 0$, which no longer agree with the experimental data.

Nevertheless, allowing longer crystallisation times pushes the kinetics to the small $N_{\text {tot }}$ regime, and consequently pushes the saturation region to larger values of $\Lambda_{0}$. For example, Fig. 17 and Fig. 18(b) shows $t_{10}$ against $N_{t o t}$ and $\Lambda_{0}$, respectively, for $N_{q, \max }=10^{6}$ and $\eta=10^{3}$. In this regime, there is a significant reduction in $t_{10}$ in the range of $\Lambda_{0}$ induced by typical print speeds.

\section{Concluding Remarks}

We have demonstrated that sufficiently low print temperatures in FFF can produce the conditions required for flow-enhanced crystallisation. That is, the deposited filament must cool below the melting temperature $T_{M}$, whilst the polymer backbone remains stretched from the flow; if full relaxation occurs before $T<T_{M}$, then there will be no flow-induced effects on the crystal morphology. For PCL, we do not observe flow-enhanced nucleation for $T_{N} \geq$ $110^{\circ} \mathrm{C}$.

On the other hand, for $T_{N}=90^{\circ} \mathrm{C}$ flow-enhanced nucleation occurs almost instantaneously once $T<T_{M}$. Since Rouse relaxation is much faster than the crystallisation rate, crystal growth follows much later. The inter-play between surface cooling and residual polymer stretch results in a boundary layer of flow-induced nuclei near to the free surface of the deposited filament, which accelerate crystal growth in this region. The bulk of the filament, on the other hand, is governed by quiescent kinetics and crystallises much later. The flow-enhanced boundary layer contains orders of magnitude more nuclei than the centre of the filament. Thus, the spherulites that grow at the surface of a deposited filament are significantly smaller than those that grow in the bulk. The thickness of this small-spherulite boundary layer is determined by thermal diffusivity.

Depth-averaging of the crystallisation time is required to compare the model predictions to a Raman spectroscopy measurement. This analysis provides a deeper understanding of the two-stage growth observed in the transient crystallisation curves. In particular, the 'step' indicates a transition from the early surface contribution to the depthaveraged crystallinity measurement, to the bulk contribution, which enters the growth later due to the slower quiescent kinetics. The model is in quantitative agreement with the experiments by tuning a single model parameter $\eta$.

We derived an analytic solution to predict $t_{10}$ at the surface under isothermal conditions such that $t_{10} \sim N_{t o t}^{-1 / 3}$. On the other hand, the typical FFF surface cooling changes the dependence of $t_{10}$ to a weaker power law closer to $N_{\text {tot }}^{-1 / 9}$. This asymptotic behaviour yields a regime where only a small degree of stretch is required for a steep decrease in $t_{10}$, yet where additional stretch $\left(\Lambda_{0}>1.1\right)$ has little effect on $t_{10}$ i.e. we observe a saturation of the flow effects for increasing stretch. This regime occurs when a large number of quiescent nuclei are created, as is the case for PCL. In this 'large $N_{t o t}$ regime', further stretching creates more nuclei at the free surface leading to the growth of smaller spherulites, however this change in morphology is not evident from a measurement of $t_{10}$. In general, if $t_{10}$ is known in the absence of flow our model can be used to predict $N_{q, \max }$ and thus determine which regime flowenhanced kinetics will reside.

We propose that flow-enhanced crystallisation can be exploited to enhance the mechanical strength at filamentfilament interfaces in FFF-printed parts. In particular, there is evidence to suggest an increase in the percent crystallinity, $\xi_{\max }$, with increasing flow rate, provided that the flow rate is faster than the slowest relaxation time $[57,58]$. Thus, due to better space filling near the free surface, flow-enhanced crystallisation can increase the availability of spherulites to form tie-chains across the weld interface. 
These tie chains are polymer chains that become anchored into crystalline structures on either side of the weld line and eliminate chain-pull-out as a failure mechanism; either the crystal structure or the polymer chain must be broken for fracture. It also seems plausible that an increased nucleation density at the surface will lead to an increased spherulite surface area close to the weld interface, ultimately leading the more of these tie-chains. Consequently, experiments of weld strength as a function of flow-enhanced crystallisation, as well as accurate models of the nucleation process, are essential.

Finally, PCL presents a special case where the polymer remains above $T_{g}$ during the printing process. Thus, nucleation is not affected by the glass transition and crystallisation proceeds to full space filling and ultimately a cross-sectionally uniform degree of crystallinity. However, for other semi-crystalline printing materials such as polylactic acid (PLA), the glass transition may arrest crystallisation and lead to a non-uniform crystal morphology across the layer. Consequently, even a single deposited filament may exhibit great spatial-variability in mechanical strength. We will investigate this effect in future publications.

\section{Acknowledgments}

The authors thank Anthony Kotula, Jonathan Seppala, and Kalman Migler for providing data, for helpful discussions and an enjoyable collaboration. CM is grateful to the Royal Commission for the Exhibition of 1851 for funding. RSG gratefully acknowledges funding from the EPSRC (grant no. EP/P005403/1)

\section{Appendix A. Latent Heat Effects}

At the onset of crystal growth $\partial T / \partial t \rightarrow 0$. Thus, Eq. 32 becomes

$$
\alpha \frac{1}{r} \frac{\partial}{\partial r}\left(r \frac{\partial T}{\partial r}\right)=\frac{H_{f}}{C_{p}} \dot{\xi} .
$$

For latent heat effects to be evident, the right-hand side must dominant. Thus, via a scaling argument we have

$$
\frac{H_{f}}{C_{p}} \dot{\xi}>\frac{\alpha T_{a}}{R_{N}^{2}} \Rightarrow \dot{\xi}>\frac{C_{p} \alpha T_{a}}{H_{f} R_{N}^{2}},
$$

which from the properties listed in Table 1 yields $\dot{\xi}>$ $8.5 \times 10^{-2} \mathrm{~s}^{-1}$. However, we have seen for $T_{N}=110^{\circ} \mathrm{C}$ quiescent crystal growth onsets approximately $20 \mathrm{~s}$, and reaches $10 \%$ crystallinity at $35 \mathrm{~s}$; a linear approximation yields $\dot{\xi}=6.7 \times 10^{-3}$. Consequently, latent heat effects can be assumed small compared to thermal diffusivity.

\section{Appendix B. Linear Rheology}

The relaxation times of the printing material are calculated by fitting the time-temperature superposed linear

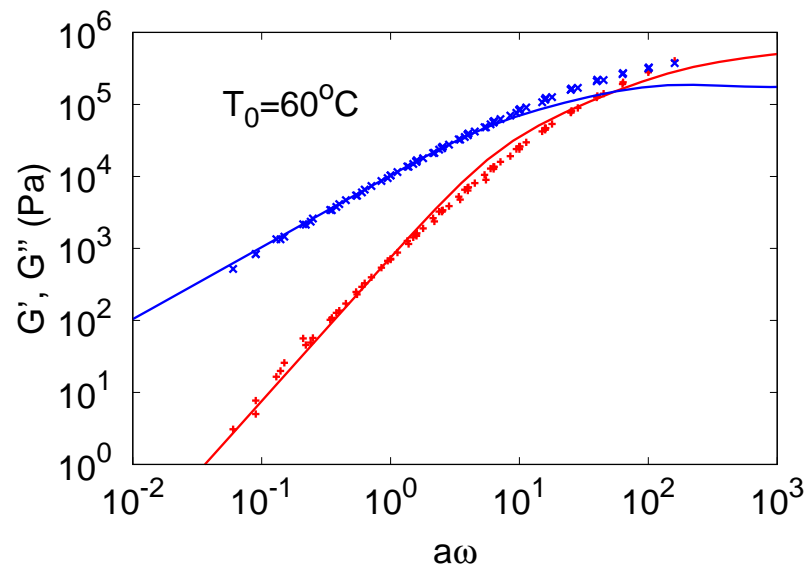

Figure B.1: Storage and loss moduli $\left(G^{\prime}, G^{\prime \prime}\right)$ plotted against the normalised frequency $a \omega$ and corresponding fit to the Likhtman \& McLeish model [33] to calculate the entanglement molecular weight $M_{e}$, the plateau modulus $G_{e}$, and the entanglement time $\tau_{e}^{0}$.

rheology data $\left(G^{\prime}, G^{\prime \prime}\right)$ to the Likhtman \& McLeish model [33], and is executed using Reptate software [34]. The fit is shown in Fig. B.1.

\section{Appendix C. Nucleation Rate}

Since the units quoted for nucleation rate in Table 1 of Ref. [36] are arbitrary, we assume that the initial nucleation rate per cubic micron per minute is given by

$$
\dot{N}_{1}=D \dot{N}_{1}^{C h y} \quad \mu^{-3} \min ^{-1}
$$

where $D$ is some constant that converts the value quoted in Ref. [36], denoted $\dot{N}_{1}^{C h y}$, to $\mu^{-3} \min ^{-1}$. The constant $D$ is then chosen to replicate Figs. 4 and 5 in [36], where the following equations (Eqs 3,5 and 8 in [36]) are plotted:

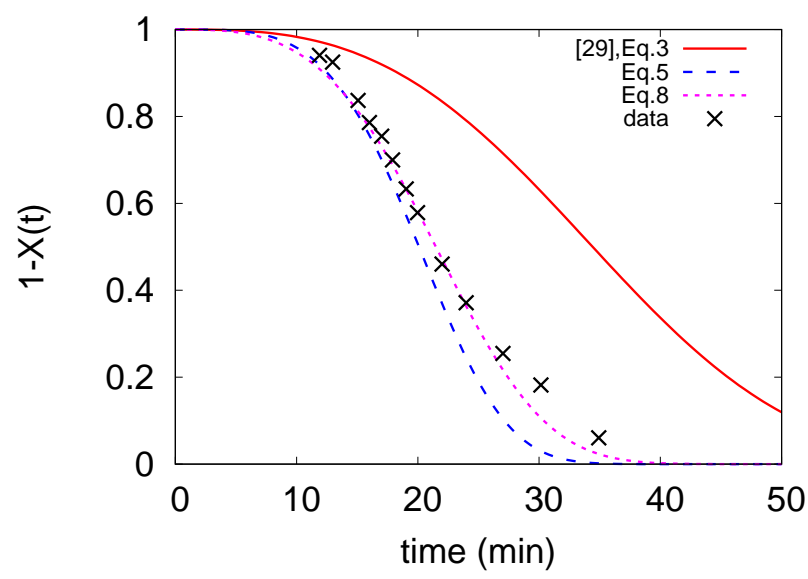

Figure C.2: Points: Fraction of untransformed PCL $1-X(t)$ measured using dilatometry at $T=46.5^{\circ} \mathrm{C}$ reproduced from Fig.5a in Ref. [36]. Lines: Eqs. (C.2)(a-c) with nucleation rate $\dot{N}_{1}$ given by Eq. C. 1 with $D=5 \times 10^{-7}$ to be compared with Eqs. 3,5 and 8 in Ref. [36]. 


$$
\begin{aligned}
& 1-X(t)=\exp \left(-\frac{\pi}{3} \frac{\rho_{s}}{\rho_{l}} \dot{N}_{1} G^{3} t^{4}\right), \\
& 1-X(t)=\exp \left(-\frac{4 \pi}{3} \frac{\rho_{s}}{\rho_{l}} \dot{N}_{1} G^{3} t^{3}\right), \\
& 1-X(t)=\exp \left(-\frac{\pi}{3} \frac{\rho_{s}}{\rho_{l}} G^{3}\left(\dot{N}_{1} t_{c}+\dot{N}_{2} t-\dot{N}_{2} t_{c}\right) t^{3}\right) .
\end{aligned}
$$

Here $X(t)$ is mass fraction transformed to solid, $\rho_{s}$ and $\rho_{l}$ are the solid and molten densities, and $t_{c}$ is the time at which the nucleation rate changes. Fig. C.2 shows the curves obtained using $D=5 \times 10^{-7}$ compared to the experimental data for $T=46.5^{\circ}$. The corresponding initial nucleation rates for each temperature are given in Table C.1. The same conversion is applied to $\dot{N}_{2}$.

Table C.1: Initial nucleation rate $\dot{N}_{1}$ for isothermal crystallisation temperatures converted to appropriate units.

\begin{tabular}{c|c|c|c}
\hline \hline Temp $\left({ }^{\circ} \mathrm{C}\right)$ & $\dot{N}_{1}^{C h y}(-)$ & $\dot{N}_{1}\left(\mu^{-3} \mathrm{~min}^{-1}\right)$ & $\dot{N}_{1}\left(\mathrm{~m}^{-3} \mathrm{~s}^{-1}\right)$ \\
\hline \hline 46.5 & 4 & $2 \times 10^{-6}$ & $3.3 \times 10^{10}$ \\
47.6 & 1.4 & $7 \times 10^{-7}$ & $1.16 \times 10^{10}$ \\
49.4 & 0.4 & $2 \times 10^{-7}$ & $3.3 \times 10^{9}$ \\
51.0 & 0.23 & $1.15 \times 10^{-7}$ & $1.9 \times 10^{9}$ \\
\hline
\end{tabular}

\section{Appendix D. Crystallisation Curves}

Fig. D. 3 compares the degree of crystallinity measured over time via in-situ Raman spectroscopy [30] to that predicted by the model for two different print temperatures $T_{N}=110$ and $140^{\circ} \mathrm{C}$, and therefore temperature histories. Note that measurements for three different speeds are shown for each print temperature, indicating there are no flow effects on the crystallisation time for $T_{N} \geq 110^{\circ} \mathrm{C}$. In these cases, the temperature of the filament is uniform once $T<T_{M}$, so that nucleation occurs uniformly across the filament and depth-averaging also does not affect predicted crystallisation times.

We find that even though the growth rate and nucleation rate must be extrapolated to low temperatures (Fig. 4), the model is in agreement with the experimental data. Crystallisation proceeds to full space filling $\xi_{g}=1$, with the maximum degree of crystallinity reaching $\xi_{\max }=0.4$. We note there is some discrepancy for $\xi>0.1$ that may need to be considered if we were interested in times greater than $t_{10}$. Furthermore, the saturation limit $N_{q, \max }=10^{12}$ is able to capture both temperature histories, indicating that saturation is independent of temperature.

As shown earlier in the main text, Fig. 9 shows the degree of crystallinity measured over time via in-situ Raman spectroscopy [30] for print temperature $T_{N}=90^{\circ} \mathrm{C}$, where FFF-flow-enhanced nucleation occurs. The depthaveraged crystallinity predicted by the model is in qualitative agreement with the experimental data, and reveals the
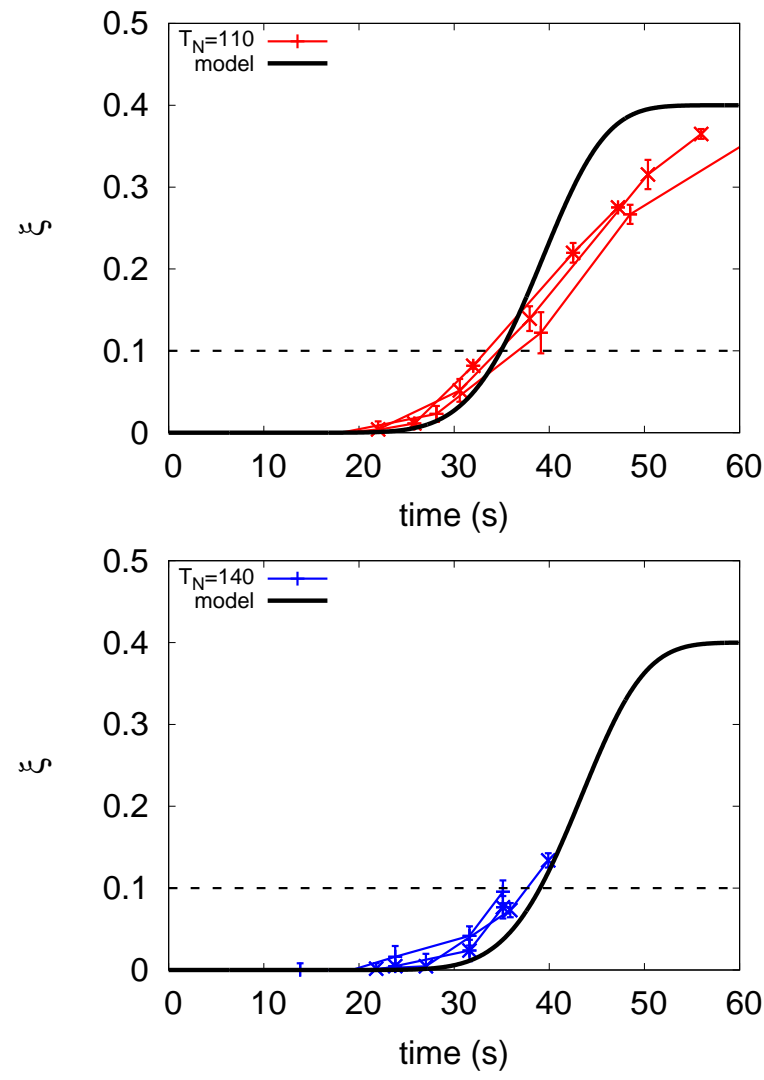

Figure D.3: Evolution of the degree of crystallinity $\xi$ measured using Raman spectroscopy and predicted by the model for $T_{N}=110$ and $140^{\circ} \mathrm{C}$, where flow-enhanced crystallisation is absent and $\xi(x, z, t)=$ $\xi_{a v}$.

mechanism governing the 'stepping' behaviour in the crystal evolution. We suspect there is some quantitative discrepancies arising from the calculation of the initial stretch profile.

The limiting assumptions of the flow model used for calculating $\Lambda_{0}$ for a particular print speed are detailed in Ref. [12]. In particular, the model assumes a steady state flow, neglects shear heating in the nozzle, and assumes that deposition occurs faster than polymer relaxation. Furthermore, since in this model crystallisation only depends on the degree of stretch and not the entanglement network, a flow-dependent entanglement number (as incorporated in Ref. [12]) has been omitted; including disentanglement during the nozzle and deposition flow calculations would also change the value of $\Lambda_{0}$. Discrepancies may also arise from approximating a distribution of molecular weights (poly-dispersity) via a single mode constitutive model.

\section{Appendix E. Isothermal Conditions}

Consider the case where the temperature is fixed at $T=40^{\circ} \mathrm{C}$. Fig. E. 4 shows the corresponding nucleation and crystal growth curves at positions $\mathrm{a}, \mathrm{b}$ and $\mathrm{m}$ under these isothermal conditions. Polymers at $\mathrm{a}, \mathrm{b}$ and $\mathrm{m}$ nucleate at $t=0$ since $T<T_{M}, \forall t$ and nucleation ceases once 

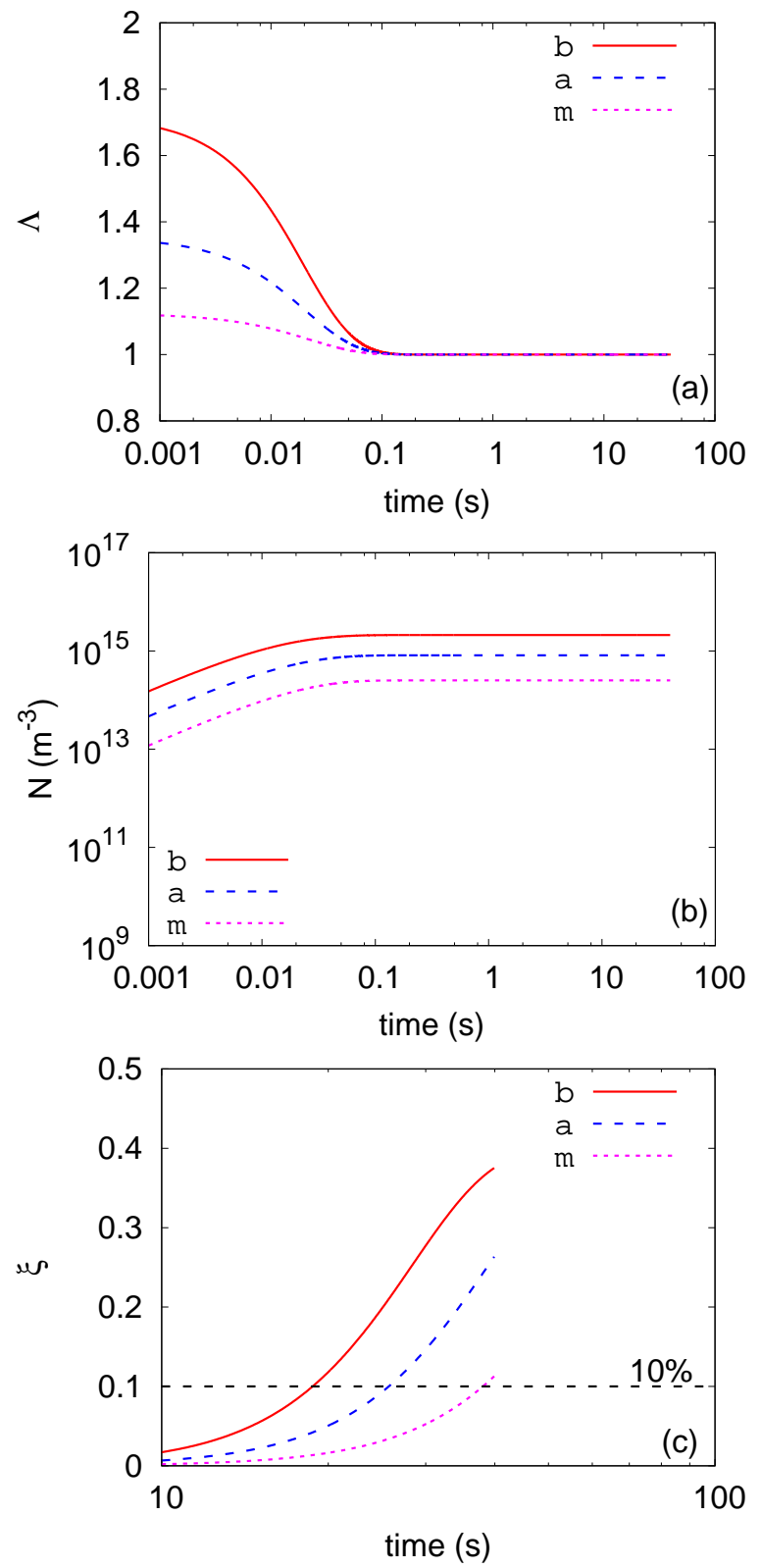

Figure E.4: (a) Degree of stretch $\Lambda$, (b) number of nuclei $N$, and (b) degree of crystallinity $\xi$ over time, at the surface $a, b$ and the centre m of the deposit under isothermal conditions $T=40^{\circ} \mathrm{C}, \forall t$ and print speed $U_{N}=30 \mathrm{~mm} / \mathrm{s}$ (which yields stress evolution similar to Fig. $14(\mathrm{a}))$. We set $\eta=10^{4}$ to give crystallisation times similar to nonisothermal case.

the stretch has relaxed. Note that this fixed temperature profile changes the stretch evolution profile very little from Fig. 14(a), with the stretch fully relaxing homogeneously across the deposit and a little later (around $\sim t=0.1 \mathrm{~s}$ ) (Fig. E.4(a)).

The difference between $t_{10}$ at $\mathrm{b}$ and $\mathrm{a}$ is significantly larger than that found in the non-isothermal case (Fig. 14). To highlight this isothermal surface crystallisation, Fig. E.5 shows $t_{10}$ as a function of the total number of nuclei $N_{\text {tot }}$ and the initial stretch $\Lambda_{0}$. We show results for two print speeds $U_{N}=20$ and $30 \mathrm{~mm} / \mathrm{s}$; the two points
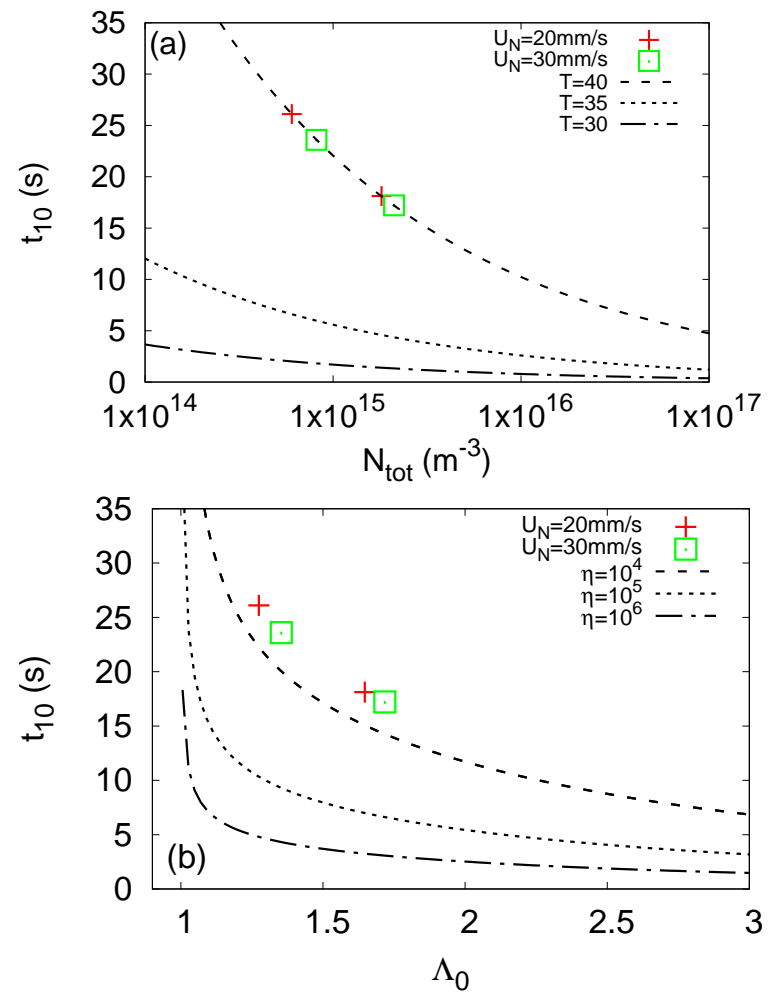

Figure E.5: Time to $10 \%$ crystallisation $t_{10}$ at the surface plotted against (a) the total number of nuclei $N_{\text {tot }}$ and (b) the initial stretch $\Lambda_{0}$ for isothermal conditions $T=40^{\circ} \mathrm{C}$, and print speeds $U_{N}=$ 20 and $30 \mathrm{~mm} / \mathrm{s}$. The two points for each speed represent surface positions a,b. The lines show the effect that changing $T$ and $\eta$ in Eq. (45) has on the analytic solution (40).

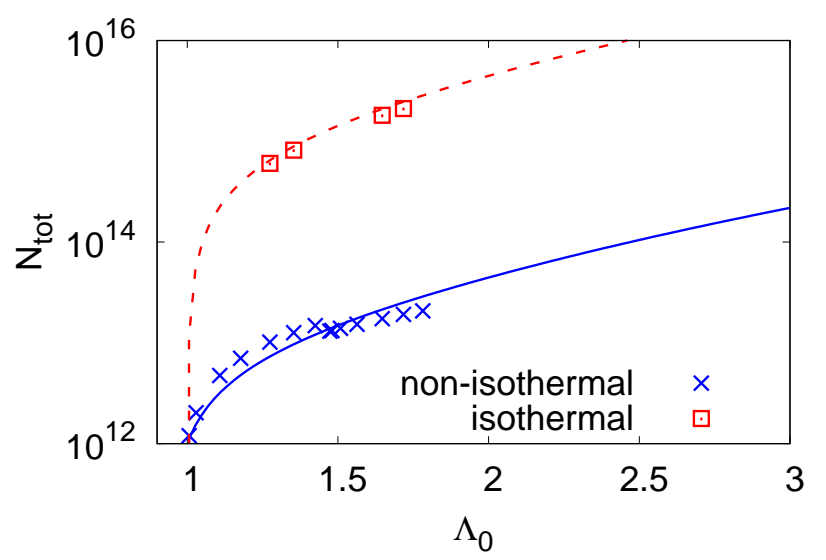

Figure E.6: Total number of nuclei created $N_{\text {tot }}$ plotted against initial stretch $\Lambda_{0}$ for isothermal $\left(T=40^{\circ} \mathrm{C}, \eta=10^{4}\right)$ and nonisothermal conditions $\left(T \equiv T(t), \eta=10^{9}\right)$ described in the text, compared to Eq. (45).

for each speed corresponding to surface positions a and $\mathrm{b}$. We set $\eta$ to $10^{4}$ to limit the total number of nuclei created so that $t_{10} \sim 30 \mathrm{~s}$, similar to non-isothermal conditions.

In this case the saturation effect seen in the equivalent non-isothermal Fig. 16(b) is absent in the range $1<\Lambda_{0}<3$, but can be achieved by pushing the kinetics to the large $N_{\text {tot }}$ regime, as shown in Fig. E.5(b); that 
is, by increasing the tuning parameter $\eta$ in Eq. (45), $t_{10}$ also becomes relatively constant with increasing $\Lambda_{0}$ in the range $1.1<\Lambda_{0}<3$.

Finally, Fig. E.6 shows the analytical solution predicting how the total number of nuclei $N_{t o t}$ depend on stretch (Eq. 45) for both the isothermal case (with $\eta=10^{4}$ ) and the non-isothermal case (with $\eta=10^{9}$ ).

[1] B. N. Turner, R. Strong, S. A. Gold, A review of melt extrusion additive manufacturing processes: I. Process design and modeling, Rapid Prototyping Journal 20 (3) (2014) 192-204.

[2] S.-H. Ahn, M. Montero, D. Odell, S. Roundy, P. K. Wright, Anisotropic material properties of fused deposition modeling ABS, Rapid Prototyping Journal 8 (4) (2002) 248-257.

[3] S. H. Ahn, C. Baek, S. Lee, I. S. Ahn, Anisotropic tensile failure model of rapid prototyping parts-fused deposition modeling (FDM), International Journal of Modern Physics B 17 (08n09) (2003) 1510-1516.

[4] C. Lee, S. Kim, H. Kim, S. Ahn, Measurement of anisotropic compressive strength of rapid prototyping parts, Journal of materials processing technology 187 (2007) 627-630.

[5] Q. Sun, G. Rizvi, C. Bellehumeur, P. Gu, Effect of processing conditions on the bonding quality of FDM polymer filaments, Rapid Prototyping Journal 14 (2) (2008) 72-80.

[6] I. Gibson, D. W. Rosen, B. Stucker, et al., Additive manufacturing technologies, Springer, New York, 2010.

[7] A. Arivazhagan, S. Masood, Dynamic mechanical properties of ABS material processed by fused deposition modelling, Int J Eng Res Appl 2 (3) (2012) 2009-2014.

[8] A. K. Sood, R. K. Ohdar, S. S. Mahapatra, Experimental investigation and empirical modelling of FDM process for compressive strength improvement, Journal of Advanced Research 3 (1) (2012) 81-90.

[9] D. Croccolo, M. De Agostinis, G. Olmi, Experimental characterization and analytical modelling of the mechanical behaviour of fused deposition processed parts made of ABS-M30, Computational Materials Science 79 (2013) 506-518.

[10] S.-Q. Wang, Nonlinear rheology of entangled polymers at turning point, Soft Matter 11 (2015) 1454-1458, doi: 10.1039/C4SM02664K.

[11] F. Snijkers, R. Pasquino, P. D. Olmsted, D. Vlassopoulos, Perspectives on the viscoelasticity and flow behavior of entangled linear and branched polymers, Journal of Physics: Condensed Matter 27 (47) (2015) 473002.

12] C. McIlroy, P. D. Olmsted, Deformation of an Amorphous Polymer during the Fused-Filament-Fabrication Method for Additive Manufacturing, Journal of Rheology 61 (2017) 379-397.

[13] A. Gleadall, I. Ashcroft, J. Segal, VOLCO: A predictive model for 3D printed microarchitecture, Additive Manufacturing .

[14] H. Xia, J. Lu, G. Tryggvason, Fully Resolved Numerical Simulations of Fused Deposition Modeling. Part II-Solidification, Residual Stresses, and Modeling of the Nozzle, arXiv preprint arXiv:1711.07094 .

[15] A. Ahmed, L. Susmel, Additively Manufactured PLA under static loading: strength/cracking behaviour vs. deposition angle, Procedia Structural Integrity 3 (2017) 498-507.

[16] M. Doi, S. F. Edwards, The theory of polymer dynamics, Oxford University Press, Oxford, 1988.

[17] T. A. Hunt, B. Todd, Diffusion of linear polymer melts in shear and extensional flows, The Journal of chemical physics 131 (5) (2009) 054904.

[18] T. Uneyama, K. Horio, H. Watanabe, Anisotropic mobility model for polymers under shear and its linear response functions, Physical Review E 83 (6) (2011) 061802.

[19] P. Ilg, M. Kröger, Molecularly derived constitutive equation for low-molecular polymer melts from thermodynamically guided simulation, Journal of Rheology 55 (1) (2011) 69-93.

[20] Y.-Q. Xue, T. Tervoort, P. Lemstra, Welding behavior of semicrystalline polymers. 1 . The effect of nonequilibrium chain conformations on autoadhesion of UHMWPE, Macromolecules
31 (9) (1998) 3075-3080.

[21] H. Zuidema, G. W. M. Peters, H. E. H. Meijer, Development and Validation of a Recoverable Strain-Based Model for FlowInduced Crystallization of Polymers, Macromolecular Theory and Simulations 10 (5) (2001) 447-460.

[22] T. Deplancke, O. Lame, F. Rousset, I. Aguili, R. Seguela, G. Vigier, Diffusion versus cocrystallization of very long polymer chains at interfaces: experimental study of sintering of UHMWPE nascent powder, Macromolecules 47 (1) (2013) 197207.

[23] E. SACHLOS, Making tissue engineering scaffolds work, Eur. Cell. Mater. 5 (2003) 29-40.

[24] J. Long, H. Gholizadeh, J. Lu, C. Bunt, A. Seyfoddin, Application of fused deposition modelling (FDM) method of 3D printing in drug delivery, Current pharmaceutical design 23 (3) (2017) 433-439.

[25] G. Jarrousse, Self adhesion of semi-crystalline polymers between their glass transition temperature and their melting temperature, Ph.D. thesis, Université Pierre et Marie Curie-Paris VI, 2004.

[26] V. Srinivas, C. S. van Hooy-Corstjens, J. A. Harings, Correlating molecular and crystallization dynamics to macroscopic fusion and thermodynamic stability in fused deposition modeling; a model study on polylactides, Polymer 142 (2018) 348-355.

[27] L. Wang, W. M. Gramlich, D. J. Gardner, Improving the impact strength of Poly (lactic acid)(PLA) in fused layer modeling (FLM), Polymer 114 (2017) 242-248.

[28] B. Wittbrodt, J. M. Pearce, The effects of PLA color on material properties of 3-D printed components, Additive Manufacturing 8 (2015) 110-116.

[29] R. S. Graham, Modelling flow-induced crystallisation in polymers, Chem. Commun. 50 (2014) 3531-3545, doi: 10.1039/C3CC49668F.

[30] L. A. Northcutt, S. V. Orski, K. B. Migler, A. P. Kotula, Effect of processing conditions on crystallization kinetics during materials extrusion additive manufacturing, Polymer

[31] C. McIlroy, P. D. Olmsted, Disentanglement Effects on Welding Behaviour of Polymer Melts during the Fused-FilamentFabrication Method for Additive Manufacturi, Polymer 123 (2017) 376-391.

[32] D. W. Hutmacher, T. Schantz, I. Zein, K. W. Ng, S. H. Teoh, K. C. Tan, Mechanical properties and cell cultural response of polycaprolactone scaffolds designed and fabricated via fused deposition modeling, Journal of Biomedical Materials Research: An Official Journal of The Society for Biomaterials, The Japanese Society for Biomaterials, and The Australian Society for Biomaterials and the Korean Society for Biomaterials 55 (2) (2001) 203-216.

[33] A. E. Likhtman, T. C. McLeish, Quantitative theory for linear dynamics of linear entangled polymers, Macromolecules 35 (16) (2002) 6332-6343.

[34] A. Likhtman, Reptate, https://reptate.com, 2009.

[35] M. L. Williams, R. F. Landel, J. D. Ferry, The temperature dependence of relaxation mechanisms in amorphous polymers and other glass-forming liquids, Journal of the American Chemical society 77 (14) (1955) 3701-3707.

[36] K. Chynoweth, Z. Stachurski, Crystallization of poly(caprolactone), Polymer 27 (12) (1986) 1912 - 1916, ISSN 00323861.

[37] G. W. Peters, L. Balzano, R. J. Steenbakkers, Flow-induced crystallization, Handbook of polymer crystallization (2013) 399-431.

[38] M. Avrami, Kinetics of Phase Change. II TransformationTime Relations for Random Distribution of Nuclei, The Journal of Chemical Physics 8 (2) (1940) 212-224, doi:10.1063/1.1750631.

[39] M. Labet, W. Thielemans, Synthesis of polycaprolactone: a review, Chemical Society Reviews 38 (12) (2009) 3484-3504.

[40] P. Skoglund, Å. Fransson, Continuous cooling and isothermal crystallization of polycaprolactone, Journal of Applied Polymer Science 61 (13) (1996) 2455-2465.

[41] N. Tsutsumi, K. Kano, Thermal diffusivity of polycaprolac- 
tone/poly (bisphenol A carbonate) blends by flash radiometry, Journal of Polymer Science Part B: Polymer Physics 37 (8) (1999) 745-749.

[42] J. I. Lauritzen Jr, J. D. Hoffman, Extension of theory of growth of chain-folded polymer crystals to large undercoolings, Journal of applied Physics 44 (10) (1973) 4340-4352.

[43] M. E. Mackay, Z. R. Swain, C. R. Banbury, D. D. Phan, D. A. Edwards, The performance of the hot end in a plasticating 3D printer, Journal of Rheology 61 (2) (2017) 229-236.

[44] B. Robertson, R. L. Thompson, T. C. McLeish, I. Robinson, Theoretical prediction and experimental measurement of isothermal extrudate swell of monodisperse and bidisperse polystyrenes, Journal of Rheology 61 (5) (2017) 931-945.

[45] O. Carneiro, A. Silva, R. Gomes, Fused deposition modeling with polypropylene, Materials \& Design 83 (2015) 768-776.

[46] R. Comminal, M. P. Serdeczny, D. B. Pedersen, J. Spangenberg, Numerical modeling of the strand deposition flow in extrusion-based additive manufacturing, Additive Manufacturing 20 (2018) 68 - 76, ISSN 2214-8604

[47] J. E. Seppala, K. D. Migler, Infrared thermography of welding zones produced by polymer extrusion additive manufacturing, Additive Manufacturing 12 (2016) 71-76.

[48] R. Yardimici, T. Hattori, S. Guceri, S. Danforth, Thermal analysis of fused deposition modelling, in: D. Bourell, J. Beaman, H. Marcus, R. Crawford, J. Barlow (Eds.), Solid Freeform Fabrication Proceedings, University of Texas, Texas, 1997.

[49] A. DAmico, A. M. Peterson, An adaptable FEA simulation of material extrusion additive manufacturing heat transfer in $3 \mathrm{D}$, Additive Manufacturing 21 (2018) 422-430.

[50] J. Du, Z. Wei, X. Wang, J. Wang, Z. Chen, An improved fused deposition modeling process for forming large-size thin-walled parts, Journal of Materials Processing Technology 234 (2016) $332-341$.

[51] A. E. Likhtman, R. S. Graham, Simple constitutive equation for linear polymer melts derived from molecular theory: Rolie-Poly equation, Journal of Non-Newtonian Fluid Mechanics 114 (1) (2003) $1-12$.

[52] R. S. Graham, A. E. Likhtman, T. C. McLeish, S. T. Milner, Microscopic theory of linear, entangled polymer chains under rapid deformation including chain stretch and convective constraint release, Journal of Rheology 47 (5) (2003) 1171-1200.

[53] R. Graham, Molecular modelling of entangled polymers under flow, Ph.D. thesis, University of Leeds, 2002.

[54] R. S. Graham, P. D. Olmsted, Coarse-Grained Simulations of Flow-Induced Nucleation in Semicrystalline Polymers, Phys. Rev. Lett. 103 (2009) 115702, doi: 10.1103/PhysRevLett.103.115702.

[55] F. J. M. F. Custdio, R. J. A. Steenbakkers, P. D. Anderson, G. W. M. Peters, H. E. H. Meijer, Model Development and Validation of Crystallization Behavior in Injection Molding Prototype Flows, Macromolecular Theory and Simulations 18 (9) (2009) 469-494, ISSN 1521-3919, doi:10.1002/mats.200900016.

[56] R. J. A. Steenbakkers, G. W. M. Peters, A stretch-based model for flow-enhanced nucleation of polymer melts, Journal of Rheology 55 (2) (2011) 401-433, doi:10.1122/1.3545844.

[57] M. Chellamuthu, D. Arora, H. H. Winter, J. P. Rothstein, Extensional flow-induced crystallization of isotactic poly-1-butene using a filament stretching rheometer, Journal of Rheology 55 (4) (2011) 901-920.

[58] E. E. B. White, H. H. Winter, J. P. Rothstein, Extensional-flowinduced crystallization of isotactic polypropylene, Rheologica acta 51 (4) (2012) 303-314. 